Annuaire suisse de politique de développement

18 | 1999

La Suisse et l'action humanitaire

\title{
5. Politique intérieure et extérieure
}

Rosita Fibbi, Gertrud Ochsner et Nicolas Schwab

\section{CpenEdition}

\section{Journals}

Édition électronique

URL : http://journals.openedition.org/aspd/699

DOI : 10.4000/aspd.699

ISSN : 1663-9669

\section{Éditeur}

Institut de hautes études internationales et du développement

\section{Édition imprimée}

Date de publication : 1 janvier 1999

Pagination : 165-194

ISSN : 1660-5934

\section{Référence électronique}

Rosita Fibbi, Gertrud Ochsner et Nicolas Schwab, « 5. Politique intérieure et extérieure », Annuaire suisse de politique de développement [En ligne], 18| 1999, mis en ligne le 16 juillet 2012, consulté le 08 septembre 2020. URL : http://journals.openedition.org/aspd/699; DOI : https://doi.org/10.4000/aspd. 699 


\section{POLITIQUE INTÉRIEURE ET EXTÉRIEURE*}

\subsection{POLITIQUE D'ASILE ET QUESTIONS MIGRATOIRES}

Les questions migratoires ont encore une fois en 1998 jalonné l'actualité politique et médiatique en Suisse. L'article passe en revue ces évolutions en s'appuyant sur un large éventail de la presse francophone et germanophone.

La politique d'asile a nettement dominé la scène publique avec des questions relatives à la définition du cadre normatif, aux modalités de gestion de la politique d'asile et finalement aux données du phénomène de l'asile, changeantes au gré des tensions politiques internationales. Au niveau du cadre général, la refonte de la loi sur l'asile a largement occupé les Chambres fédérales jusqu'à son approbation en juin 1998; son entrée en vigueur est toutefois partiellement retardée par le lancement d'un référendum. L'arrêté fédéral urgent visant à contourner ce retard fait également l'objet d'une opposition référendaire qui toutefois n'a pas un effet suspensif. Au niveau de la mise en æeuvre de la politique de l'asile, le lancement d'une politique active de retour, reposant sur un double dispositif incitatif à l'attention des populations concernées et des zones de destination, a inauguré une collaboration entre l'ODR et la DDC. Au niveau des caractéristiques du phénomène, l'augmentation des demandes d'asile déposées suite à l'intensification de la guerre au Kosovo fait supposer que l'année 1998 renouera avec les chiffres importants enregistrés au début de la décennie.

Les récents développements en matière de politique de migration paraissent moins chargés de passion - mais pas consensuels pour autant. En ce qui concerne la politique d'admission, l'année 1998 pose officiellement un terme à la hiérarchisation stricte en fonction des pays de provenance du recrutement de la main-d'œuvre étrangère (modèle des trois cercles de 1991); en ce qui concerne la politique envers les étrangers résidents, l'inscription dans la loi de la volonté de promouvoir l'intégration des étrangers fait désormais de cet objectif une tâche relevant aussi des autorités fédérales. La claire mobilisation des autorités aux divers échelons de l'édifice publique helvétique laisse présager d'importants développements dans un proche futur.

\subsubsection{POLITIQUE D'ASILE}

\section{$\square$ Demandes d'asile déposées en 1997}

23'982 personnes ont demandé l'asile en Suisse entre janvier et décembre 1997: cette augmentation de $33 \%$ par rapport à l'année précédente contraste avec la relative stabilisation observée dans les trois années précédentes. La hausse du nombre de demandes d'asile se poursuit en 1998: entre janvier et août 21'378 nouvelles demandes ont été déposées (ce qui représente une augmentation de

\footnotetext{
Partie 5.1.: par Rosita Fibbi, sociologue, Institut d'anthropologie et de sociologie, Lausanne. Partie 5.2.: par Gertrud Ochsner, membre de l'Action Place financière Suisse à Bâle.

Partie 5.3.: par Nicolas Schwab, licencié de l'Institut universitaire des hautes études internationales (HEI) Genève.
} 
$51 \%$ par rapport à la période correspondante en 1997) ${ }^{1}$ et l'on avance le chiffre de 30'000, voire 40'000 demandes pour l'ensemble de l'année 1998.

\section{Graphique n`3: Evolution des demandes d’asile, 1987-1997}

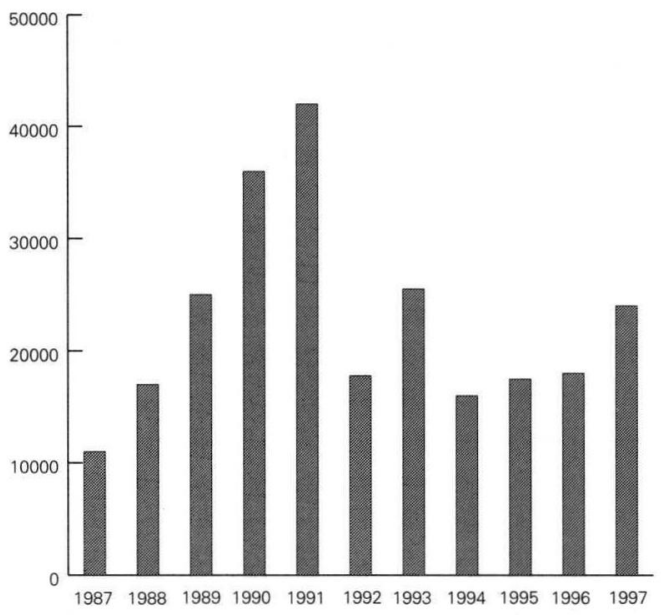

La moitié des requérants d'asile provient de la région balkanique $(49,9 \%$ contre $43 \%$ en 1996): 6913 (soit 28.8\%) sont originaires d'ex-Yougoslavie, 3081 (soit $12,8 \%$ ) d'Albanie et 1987 (soit 8,3\%) de Bosnie-Herzégovine; l'augmentation des personnes en provenance d'Albanie étant particulièrement frappante (multiplication par un facteur de 9.8). Des effectifs de demandeurs d'asile en nombres importants proviennent en outre du Sri Lanka (2137, soit 8,9\%), de Turquie (1395, soit $5,8 \%)$ et de Somalie (884, soit $3,7 \%)$.

Tableau ${ }^{\circ}{ }^{12}$ : Evolution des demandes d'asile 1988-1997 selon le pays d'origine*

\begin{tabular}{|c|c|c|c|c|c|c|c|c|c|c|}
\hline Pays d'origine & 1988 & 1989 & 1990 & 1991 & 1992 & 1993 & 1994 & 1995 & 1996 & 1997 \\
\hline Afghanistan & 71 & 134 & 234 & 233 & 278 & 144 & 172 & 114 & 198 & 215 \\
\hline Albanie & & & 250 & 1038 & 546 & 2009 & 50 & 28 & 315 & 3081 \\
\hline Algérie & & & & & & 751 & 303 & 388 & 396 & 564 \\
\hline Angola & 322 & 575 & $1 ' 134$ & 796 & 96 & 542 & 1059 & 493 & 468 & 251 \\
\hline Arménie & & & & & & & & & & 163 \\
\hline Bangladesh & 195 & 491 & 665 & 593 & 222 & 122 & 286 & 188 & 170 & 290 \\
\hline Bosnie-Herzégovine & & & & & & 6878 & 3343 & 3534 & 1269 & 1987 \\
\hline Bulgarie & 30 & 90 & 681 & 217 & 27 & 25 & 17 & 33 & & \\
\hline Chili & 139 & 39 & 11 & 7 & 6 & 1 & 4 & 0 & & \\
\hline Congo (Rép. dém) & 136 & 419 & 758 & $1 ' 426$ & 677 & 362 & 276 & 320 & 695 & 605 \\
\hline Ethiopie & 131 & 204 & 306 & 394 & 94 & 76 & 100 & 142 & 174 & 254 \\
\hline Ex-Yougoslavie & 818 & 1365 & 6545 & 14205 & 6262 & 5291 & 4124 & 5491 & 6223 & 6913 \\
\hline Ghana & 113 & 383 & 450 & 852 & 137 & 66 & 15 & 15 & & \\
\hline Guinée & & & & & & & & & & 193 \\
\hline Inde & 730 & 720 & 1'828 & 886 & 38 & 21 & 48 & 156 & 201 & 203 \\
\hline Irak & 34 & 26 & 71 & 160 & 117 & 79 & 151 & 321 & 413 & 522 \\
\hline Iran & 291 & 211 & 417 & 222 & 140 & 95 & 82 & 110 & 134 & 129 \\
\hline Liban & 529 & 2477 & 5533 & 1352 & 450 & 490 & 170 & 129 & 148 & 184 \\
\hline
\end{tabular}

1. Office fédéral des réfugiés, Statistique en matière d'asile de janvier à août 1998, ODR, août 98. 


\begin{tabular}{lrrrrrrrrrr}
\hline Pays d'origine & $\mathbf{1 9 8 8}$ & $\mathbf{1 9 8 9}$ & $\mathbf{1 9 9 0}$ & $\mathbf{1 9 9 1}$ & $\mathbf{1 9 9 2}$ & $\mathbf{1 9 9 3}$ & $\mathbf{1 9 9 4}$ & $\mathbf{1 9 9 5}$ & $\mathbf{1 9 9 6}$ & $\mathbf{1 9 9 7}$ \\
\hline Nigéria & 8 & 101 & 308 & 515 & 123 & 72 & 115 & 178 & 253 & 210 \\
\hline Pakistan & 659 & 1027 & 1212 & 1339 & 631 & 410 & 420 & 437 & 483 & 448 \\
\hline Pologne & 293 & 255 & 549 & 60 & 64 & 16 & 59 & 3 & & \\
\hline Sierra Leone & & & & & & & & & & 178 \\
\hline Roumanie & 161 & 431 & $1^{\prime} 959$ & $2{ }^{\prime} 682$ & 620 & 113 & 113 & 82 & 70 & \\
\hline Somalie & 22 & 88 & 181 & 910 & 1077 & 2295 & 881 & 478 & 700 & 884 \\
\hline Sri Lanka & 1516 & 4809 & 4774 & 7349 & 2826 & 1744 & 1487 & 1024 & 1965 & 2137 \\
\hline Syrie & 89 & 115 & 239 & 179 & 146 & 80 & 44 & 57 & 83 & \\
\hline Tchécoslovaquie & 98 & 171 & 21 & 26 & 25 & 12 & 5 & 1 & 0 & \\
\hline Turquie & 9673 & 9395 & 7262 & 4324 & 1827 & 1205 & 1068 & 1293 & 1317 & 1395 \\
\hline Vietnam & 129 & 168 & 139 & 86 & 69 & 164 & 215 & 159 & 126 & 122 \\
\hline Autres & 453 & 715 & 1441 & 1731 & 1459 & 1681 & 1359 & 1664 & 2187 & 2262 \\
\hline Total & $\mathbf{1 6 7 2 6}$ & $\mathbf{2 4 4 2 5}$ & $\mathbf{3 6 0 6 8}$ & $\mathbf{4 1 5 8 4}$ & $\mathbf{1 7 9 6 0}$ & $\mathbf{2 4 7 3 9}$ & $\mathbf{1 6 1 3 4}$ & $\mathbf{1 7 0 2 1}$ & $\mathbf{1 8 0 0 1} \mathbf{2 3 9 8 2}$ \\
\hline
\end{tabular}

Source: Office fédéral des réfugiés, Statistique en matière d'asile 1997, ODR, janvier 1998.

* Ce tableau compile les données fournies par ce document publié chaque année, qui mentionne explicitement les pays fournissant des flux importants de requérants; ainsi certains pays apparaissent dans la statistique au cours des dix dernières années alors qu'ils n'y étaient pas mentionnés auparavant (cf. Albanie, Arménie, Bosnie, Guinée, Sierra Leone); de manière analogue, l'ODR renonce à publier le chiffre relatif à certains pays lorsqu'il estime que le flux en provenance de ceux-ci se tarit (cf. Bulgarie, Chili, Ghana, Pologne, Tchécoslovaquie). Le tableau reporte tous les pays mentionnés dans le but de rendre compte des changements intervenus dans la provenance des requérants d'asile au cours de dix dernières années.

90\% des requérants arrivés en Suisse y sont entrés clandestinement; 867 d'entre eux ont déposé des demandes d'entrée à la frontière et 416 demandes d'entrée ont été remises auprès de représentations suisses à l'étranger. Bien qu'il ait augmenté, le nombre de cas traités en première instance ne parvient pas à dépasser le nombre de nouvelles demandes déposées, comme cela avait lieu au cours des dernières années: les demandes en suspens en première instance sont donc en augmentation par rapport à 1996 (17'612 contre 16’380).

\section{$\square$ Octroi de l'asile}

L'asile a été octroyé à 2636 personnes (contre 2267 en 1996). Le taux moyen de reconnaissance de la qualité de réfugié s'est élevé à 12,5\% en 1997 (contre 12\% en $1996,14,9 \%$ en 1995 et $12,5 \%$ en 1994); ce taux varie sensiblement selon la provenance des requérants: il est élevé pour les Vietnamiens $(86 \%)$ et les Irakiens (69\%), moyen pour les Turcs (48\%) et les Bosniaques (31\%), mais il est faible pour les Sri Lankais (3\%).

En 1997, 8703 personnes ont vu leurs conditions de séjour définies; 2722 en tant que réfugiés (2636 plus 86 bénéficiaires de campagnes d'accueil des réfugiés décidées par le Conseil fédéral); 2860 au titre d'une admission provisoire; 485 au titre d'un permis humanitaire. Finalement, 2636 personnes ont obtenu une autorisation régulière délivrée pour d'autres raisons par la Police des étrangers.

Le nombre de personnes relevant de l'asile se montait à 136'053 à fin 1997, soit une augmentation de 4\% par rapport à 1996. 


\begin{tabular}{lrrr}
\hline & $\mathbf{3 1 . 1 2 . 1 9 9 7}$ & $\mathbf{3 1 . 1 2 . 1 9 9 6}$ & $\begin{array}{r}\text { variation } \\
\text { en \% }\end{array}$ \\
\hline Effectif total & $\mathbf{1 3 6 0 5 3}$ & $\mathbf{1 3 0 8 7 9}$ & $\mathbf{4 . 0}$ \\
\hline Réfugiés reconnus & 23652 & 22537 & 4.9 \\
\hline Cas humanitaires/ & & & 10.8 \\
\hline cas réglés par la Police des étrangers & 31132 & 28109 & -15.9 \\
\hline Admissions provisoires & 28406 & 33767 & 20.2 \\
\hline Traitement/exécution en suspens ou bloqué & 27134 & 22570 & 8.0 \\
\hline Cas en suspens en deuxième instance & 8117 & 7516 & 7.5 \\
\hline Cas en suspens en première instance & 17612 & 16380 & \\
\hline
\end{tabular}

Source: Office fédéral des réfugiés, Statistique en matière d'asile 1997, ODR, janvier 1998.

Toujours en 1997, on a enregistré 16'622 départs, un nombre en nette augmentation par rapport à l'année précédente (+60\%): 4551 personnes ont été contraintes de partir; par ailleurs, au terme de la procédure d'asile, 1730 ont été reconduites dans leur pays d'origine et 542 vers un pays tiers. Pour 9799 requérants déboutés, on ne possède pas d'indication quant à leur domicile. L'augmentation des départs réguliers est imputable en priorité au projet d'aide au retour de la Confédération en faveur des ressortissants bosniaques ( $c f$. infra).

Les dépenses de l'Office fédéral des réfugiés (ODR) se sont élevées à 1016 millions de francs (856 millions inscrits au budget et 164 millions de crédits supplémentaires).

\section{$\square$ Politique de renvoi et d'aide au retour}

Il convient de distinguer à cet égard la situation des Yougoslaves (en majorité des Kosovars) et celle des Bosniaques. Suite à la conclusion d'un accord de réadmission avec la République fédérale de Yougoslavie, l'exécution des renvois vers ce pays, bloquée depuis 1994, a pu reprendre à partir de septembre 1997: jusqu'à fin juillet 1998, 1243 personnes ont ainsi été rapatriées. Le Conseil fédéral a levé l'admission collective provisoire octroyée aux déserteurs et réfractaires en provenance d'ex-Yougoslavie.

Quant aux Bosniaques, les autorités, estimant que 1'accord de Dayton avait créé les conditions leur permettant de rentrer sans danger chez eux, ont fixé des délais de départ au 30 avril 1997 pour les personnes seules et les couples sans enfants, au 30 avril 1998 pour les familles avec enfants et les mineurs non accompagnés, et au 31 août 1998 pour les déserteurs et les réfractaires.

Pour accompagner ces mesures et promouvoir le retour volontaire des personnes chassées par la guerre, le Conseil fédéral a mis sur pied un programme d'aide au retour. Celui-ci comporte l'allocation aux candidats au retour d'une somme de $4000 \mathrm{CHF}$ par adulte et de $2000 \mathrm{CHF}$ par enfant au titre d'aide à la réintégration, ainsi qu'une allocation de $1000 \mathrm{CHF}$ par ménage pour faire face aux premiers frais. A la fin du mois d'août 1998, 9100 Bosniaques étaient rentrés au pays dans le cadre de ce programme; 3700 autres s'apprêtent à le faire, de sorte que le succès de l'opération dépasse les attentes des autorités suisses.

Le programme d'aide au retour spécifique pour les Bosniaques «Aide à la réintégration» mis au point par la Suisse est particulièrement novateur et, à ce titre, pris comme exemple à l'étranger: il comporte, en effet, à côté des aides décrites 
ci-dessus, un second volet, la Confédération consacrant des montants équivalents à ceux consentis à titre individuel pour la reconstruction des infrastructures détruites dans les régions qui accueillent les rapatriés. Aux 30,5 mio. CHF d'aides individuelles fait donc pendant un montant de 30,5 mio. CHF pour le financement de projets d'aide structurelle ${ }^{2}$. Le programme, qui visait initialement, en 1996, seulement 1000 personnes, a rencontré un accueil favorable, et ce d'autant plus que l'on a progressivement levé l'admission provisoire pour les Bosniaques. Il inaugure une collaboration inédite entre l'ODR et la DDC (Direction du développement et de la coopération), deux institutions ayant l'habitude de travailler selon des logiques différentes.

La détérioration de la situation au Kosovo a mis un frein aux renvois des Kosovars, qui ont été finalement suspendus le 12 juin 1998. Ce faisant, la Suisse s'est alignée sur la politique suivie par l'Allemagne et l'Autriche, les pays les plus directement concernés par la présence des Kosovars. La suspension des renvois n'a cependant pas impliqué l'octroi d'une admission provisoire comme cela avait été le cas pour les Bosniaques. En septembre 1998, les Kosovars ont obtenu un sursis de six mois, jusqu'en avril 1999. Afin de ne pas augmenter l'attractivité de la Suisse, les autorités s'opposent encore, fin octobre, à prononcer l'admission provisoire - qui présenterait pourtant l'avantage de ne pas engorger la procédure d'asile - demandée par les œuvres d'entraide et certains partis (radical et socialiste).

Au cours de la période sous revue, la politique d'asile a de nouveau enflammé les esprits en Suisse, après des mois d'un calme relatif. La nomination, en novembre 1997, du nouveau responsable de l'ODR en la personne de JeanDaniel Gerber, ancien représentant de la Suisse à la Banque mondiale, mettait fin à une période d'incertitude et laissait augurer une politique plus transparente, ferme mais ouverte. Cependant, débat et mobilisation se sont vite ravivés autour de quatre aspects de la politique d'asile: l'accueil des Algériens et le renvoi des requérants déboutés, d'une part, l'augmentation des demandes d'asile et la révision de la loi sur l'asile, d'autre part.

En début d'année, une vive mobilisation, surtout en Suisse romande, a interpellé les autorités tout autant quant à la frilosité de l'accueil qu'elles réservaient aux Algériens et Algériennes qu'à la rigueur des rapatriements.

Le programme d'aide au retour n'a pas fait l'unanimité: une étude, menée par l'Institut d'ethnologie de l'Université de Berne sur mandat de la DDC, en a remis certains aspects en question ${ }^{3}$. Les auteurs concluent en effet que l'objectif de la réinsertion des ex-requérants est «seulement en partie atteint», notamment pour les personnes n'ayant pas pu rentrer dans leur région d'origine. Pour les $45 \%$ de ces «réfugiés en relocation» les conditions de réintégration seraient inacceptables. C'est pourquoi les auteurs proposent que «les délais de retour pour les familles avec enfants doivent être réexaminés» ${ }^{4}$. La polémique révèle un conflit entre un objectif inscrit dans un horizon temporel bref, le retour, et un objectif à plus longue échéance, la stabilisation socio-économique des régions accueillant les rapatriés. Sur le plan international, certaines critiques émanant d'ONG met-

2. «Retour vers la précarité», dossier Un seul monde, sept. 98.

3. L'étude se fonde sur 4294 questionnaires et 185 entretiens avec des familles (582) effectués auprès des personnes rentrées en Bosnie-Herzégovine. «Evaluation des Rückkehrhilfeprogramms: Gemischte Bilanz in Bosnien-Herzegowina», Neue Zürcher Zeitung, 31.10.98.

4. «La Suisse devrait revoir les conditions de rapatriement des Bosniaques», Journal de Genève, 31.1.98. 
tent en lumière les conséquences non intentionnelles de la politique de retour des pays tels que la Suisse et l'Allemagne, l'afflux massif des réfugiés encouragé par ces pays risquant de perturber le processus de retour des minorités et d'entériner l'épuration ethnique 5 .

Il n'est pas surprenant, avec de telles prémisses, que les modalités d'exécution des renvois aient donné lieu à de vives discussions. L'attention du public s'est focalisée sur le cas des jeunes en formation: certains cantons ont pris à la lettre les délais de départ impartis par la Confédération tandis que d'autres ont préféré prolonger le séjour afin de permettre l'achèvement des formations entreprises, tenant compte des intérêts individuels des jeunes ainsi que de la contribution qu'une personne qualifiée peut fournir dans les zones accueillant les rapatriés. La mobilisation de groupes importants ainsi que de camarades de classe des jeunes candidats au rapatriement a contribué à assouplir la rigueur des renvois. L'exécution des renvois a montré des différences non négligeables d'un canton à l'autre; il s'agit d'un épisode significatif des tensions apparaissant de plus en plus ouvertement entre cantons et Confédération dans la gestion de la politique d'asile et, en général, des politiques d'intégration.

\section{$\square$ Révision de la loi sur l'asile}

La révision de la loi sur l'asile est un chantier ouvert depuis 1993, qui concerne un texte long et compliqué (117 articles). Le Conseil national a traité la question en juin et le Conseil des Etats en décembre 1997; le Conseil national a été saisi encore une fois de cette révision en mars et le Conseil des Etats à fin avril 1998 avant de l'approuver définitivement. Il ne s'agit là que des derniers épisodes d'une très longue histoire, dont on trouve une chronologie sommaire dans l'encadré ci-après. Les autorités ont désormais renoncé à leur projet d'une loi susceptible d'englober migrations économique et politique; le travail législatif actuel présente donc un certain caractère de durabilité.

\section{En guise de repères, une chronologie de la loi sur l'asile}

1981 Entrée en vigueur de la loi sur l'asile. La Suisse se conforme à la Convention internationale de Genève de 1951 qui règle le statut de réfugié et le principe de non-refoulement.

1983 Première révision de la loi. Pour que la Confédération puisse faire face aux dossiers en souffrance qui ne cessent d'augmenter, la procédure d'asile, jugée trop complexe, est allégée (notamment pour les "cas manifestement infondés"; désormais, une décision de renvoi accompagne les décisions négatives sur l'asile; par ailleurs, les requérants ne pourront plus travailler pendant les trois premiers mois de leur séjour). La loi entre en vigueur en 1984.

1986 Deuxième révision. Les retards ne sont pas résolus. Le Conseil fédéral propose un allégement ultérieur de la procédure: désormais, la prise de décision sur dossier devient la règle, l'assistance peut déroger aux normes usuelles de minimum vital (inférieure de 20\%), les réfugiés sont répartis entre les cantons sans tenir compte de leurs liens sociaux, la détention administrative en vue du refoulement est créée. Suite à un référendum, le peuple et les cantons acceptent la loi, qui entre en vigueur en 1988.

1990 La troisième révision entre en vigueur. Par un arrêté fédéral urgent la Suisse avait refusé l'entrée des ressortissants des pays définis comme sûrs. De plus, la nouvelle loi introduit une interdiction de travail systématisée pendant trois mois; un montant (7\%, porté à 10\% en 1995) pour garantir

5. «Le retour vers la précarité», Le Temps, 8.9.98 
la couverture d'assistance est prélevé sur le salaire du requérant. En guise d'ouverture, on admet les "réfugiés de la violence».

1991 Les nouvelles requêtes dépassent 41'000 unités, un record. Les Démocrates suisses lancent un référendum "Pour une politique d'asile raisonnable», qui réclame l'expulsion immédiate des requérants entrés illégalement dans le pays.

1993 L'UDC lance un référendum "Contre l'immigration illégale».

1994 Le Parlement adopte les "Mesures de contrainte» visant en premier lieu les étrangers en situation illégale identifiés comme dangereux, notamment les trafiquants de drogue. La loi introduit la détention préparatoire de l'expulsion. Un référendum est lancé contre ces mesures, qui sont toutefois approuvées en votation par $73 \%$ des Suisses. Ces mesures sont entrées en vigueur en 1995.

1995 Mise en chantier de la révision totale de la loi, visant entre autres à traduire en droit ordinaire le droit d'urgence de 1990.

1996 L'initiative des Démocrates suisses est jugée irrecevable; en décembre le peuple repousse de justesse $(53 \%)$ celle de l'UDC.

1998 Face à l'augmentation du nombre des requérants d'asile, le Conseil fédéral introduit un arrêté urgent - entré en vigueur le $1^{\text {er }}$ juillet 1998 - visant à écarter de la procédure régulière les requérants sans papier et ceux entrés illégalement. II reprend ainsi à son compte la proposition UDC qu'il avait pourtant combattue lors de la votation de décembre 1996. Un double référendum, contre la loi et contre l'arrêté fédéral urgent, est lancé contre ces mesures; il a abouti.

Sources: "Droit d'asile: quinze ans de démantèlement», Vivre ensemble, n 69, septembre 98; "Asile, la situation devient chaude», Le Temps, 17.6.98

Dans son édition de 1998, l'Annuaire résume les trois points principaux de cette révision: 1) création d'une base légale pour l'attribution d'une protection pour les personnes fuyant des régions en guerre, les «réfugiés de la violence»; 2) prise en compte des motifs spécifiques aux femmes dans la décision de leur accorder une protection; 3) non-entrée en matière pour les requérants entrés clandestinement dans le pays.

口i ASTM 1998, p. 244.

1) L'article qui sert de base à la définition du nouveau statut de protection provisoire est formulé de la manière suivante: «La Suisse peut accorder la protection provisoire à des personnes à protéger, aussi longtemps qu'elles sont exposées à un danger général grave, notamment pendant une guerre ou une guerre civile, ainsi que lors d'une situation de violence généralisée.» Ce statut fournit une protection aux personnes non menacées à titre individuel et donc non expressément couvertes par la Convention de Genève et permet de faire face à un afflux massif. Toutefois certains milieux font valoir que les personnes bénéficiant de ce nouveau statut jouissent d'une moins bonne protection que celle accordée aux réfugiés statutaires, notamment en excluant la perspective d'une intégration dans le nouveau pays de résidence. De plus, ils critiquent l'interdiction qui est faite aux personnes admises collectivement pendant cinq ans de déposer une demande d'asile individuelle.

2) Au terme d'un débat qui avait vu les deux Chambres opposées sur ce point, la loi sur l'asile dit à l'article 3 qu' «il y a lieu de tenir compte des motifs de fuite spécifiques aux femmes»; certains commentateurs attirent cependant l'attention sur la différence entre «motifs de fuite» et "motifs de persécution», ces derniers demeurant les seuls à fonder la définition du réfugié. 
3) Les Chambres ont décidé d'écarter de la procédure régulière d'asile les étrangers en situation irrégulière qui ne déposeraient pas une demande d'asile dans les dix jours et les requérants qui ne présenteraient pas leurs papiers d'identité (rappelons qu'en 1997, 90\% des demandeurs d'asile sont entrés clandestinement). Les deux nouvelles causes de non-entrée en matière ont été atténuées, afin de les rendre conformes au droit international, comme le recommandait un avis de droit du professeur Kälin, demandé par le HCR.

Au terme d'une analyse critique de l'ensemble de la loi, la Coordination asile Suisse a décidé de lancer un référendum contre cette loi, avant même la clôture des travaux parlementaires, pour porter le débat sur la place publique. Quelles sont les objections avancées? Outre la profonde insatisfaction face aux nouvelles dispositions sur la non-entrée en matière opposée aux sans-papiers et aux illégaux, les milieux hostiles à la loi contestent que celle-ci puisse être efficace face à l'augmentation des demandes et aux abus, par ailleurs considérés comme montés en épingle; ils font aussi valoir qu'il est souvent impossible de renvoyer les personnes sans papiers et sans motif d'asile. L'autre critique majeure concerne l'exclusion de la procédure d'asile pendant cinq ans des réfugiés de la violence acceptés collectivement.

En réaction au lancement de ce référendum, le Conseil fédéral a accéléré l'entrée en vigueur des nouvelles dispositions sur la non-entrée en matière par un arrêté fédéral urgent (AFU), approuvé à la mi-juin par les Chambres et entré en vigueur le $1^{\text {er }}$ juillet 1998 . Ce verrouillage de la politique d'asile de la part du gouvernement doit être situé dans le contexte de l'augmentation des demandes d'asile, dramatisé par la proposition de l'UDC d'engager l'armée contre l'immigration clandestine, proposition que le Conseil fédéral a finalement rejetée en juin ${ }^{6}$.

Les milieux attachés à la sauvegarde du droit d'asile ont dès lors décidé le lancement d'un deuxième référendum contre l'AFU. L'Organisation suisse d'aide aux réfugiés (OSAR) fait en effet valoir que, d'une part, ce sont les véritables réfugiés qui sont privés de documents d'identité, ne pouvant s'adresser aux autorités de leur pays d'origine pour l'établissement d'un passeport, et, d'autre part, que les dispositions de l'AFU recoupent sur le plan politique celles rejetées par le peuple lors de la votation de décembre 1996 (initiative contre l'immigration illégale). Les deux référendums ont abouti le 15 octobre 1998, les votations auront lieu au cours du premier semestre 1999, à quelques mois du renouvellement du Parlement national.

A la mi-octobre, on observe un revirement soudain du climat politique autour de l'asile des Kosovars. Suite à l'aggravation de la situation militaire sur place, le nombre mensuel de requérants est passé de 3300 à 5500 dans l'espace de deux mois, de sorte que l'on avance le pronostic de 40'000 demandes d'asile pour l'année 1998, un retour au record de 1991. En Suisse il n'est plus question d'abus et de sans-papiers mais de personnes dans le besoin et sans toit : il est même reproché aux autorités de ne pas avoir su augmenter à temps les capacités logistiques d'accueil des familles. Jusqu'à l'aile modérée de l'UDC, plus aucune force politique ne conteste la légitimité de la demande de protection des Kosovars. Le

6. «Compte tenu du climat actuel, qui permet d'aller jusqu'à envisager d'envoyer la troupe à la frontière, on ne s'étonnera donc pas de voir les sans-papiers et les illégaux exclus de la procédure d'asile» («Un Conseil fédéral sous influence», Le Temps, 14.5.98) 
Conseil fédéral, en appelant à la compassion de la population suisse envers les Albanais du Kosovo, décide d'employer des soldats non armés pour encadrer les requérants d'asile, et non pas pour garder les frontières.

L'ODR convoque une conférence nationale de coordination sur la politique d'asile et propose avec insistance au HCR d'en convoquer une internationale pour mieux répartir la charge de l'accueil des Kosovars.

\section{$\square$ Collaboration avec l'Union européenne et les Etats qui en sont membres}

Le $1^{\text {er }}$ septembre 1997 est entrée en vigueur la Convention de Dublin, relative à la détermination de l'Etat responsable de l'examen d'une demande d'asile présentée dans l'un des pays de l'Union européenne. La mise en œuvre opérationnelle de l'outil indispensable à l'application de la Convention de Dublin, à savoir la banque des donnés pour la comparaison des empreintes digitales EURODAC, est restée toutefois en suspens. Face à l'impossibilité d'adhérer à la convention, le Conseil fédéral souhaitait parvenir à un accord parallèle avec l'UE. Or, depuis l'automne 1997, l'UE fait dépendre cette démarche de la conclusion des négociations bilatérales et, en particulier, d'un accord sur la libre circulation des personnes.

La Suisse cherche à atténuer les inconvénients de sa non-participation à la Convention de Dublin, notamment en négociant des accords bilatéraux avec les pays limitrophes, dans le but d'assurer la réadmission par ces Etats des personnes entrées illégalement en Suisse. Pendant l'été, de tels accords ont été conclus entre autres avec la France et l'Italie. Le Parlement devra se prononcer prochainement sur ces accords. La révision et la mise à jour des accords précédemment conclus avec l'Allemagne et l'Autriche sont en cours.

\subsubsection{POLITIOUE DE MIGRATION}

La politique de migration comporte deux volets, la politique d'admission et celle à l'égard des étrangers. Le premier volet a traditionnellement occupé le devant de la scène politique suisse; par contre cette année se caractérise par une discussion renouvelée sur la politique envers les étrangers admis sur le territoire, et un virage clair en faveur d'une véritable politique d'intégration, qui a fait défaut jusqu'ici.

\section{$\square$ Politique d'admission}

La modification de l'Ordonnance annuelle limitant le nombre des étrangers (OLE) cache cette année une réforme de taille: l'abandon de la politique des trois cercles de 1991, relative aux critères et aux modalités de sélection de la maind'œuvre étrangère, vivement contestée, entre autres, par la Commission fédérale contre le racisme. «Sans s'épancher sur le caractère raciste de cette politique»? le Conseil fédéral donne ainsi une suite concrète à sa volonté de suivre la commission d'experts Hug sur une nouvelle conception de la politique en matière de migration $^{8}$,qui critiquait le recrutement de la main-d'œuvre selon des critères géographiques.

@D ASTM 1998, p. 245.

7. «Une nouvelle loi pour confirmer l'abandon de la politique des «trois cercles»», Le Temps, 9.6.98.

8. Voir le rapport sur la politique de migration publié en août 1997 (cf. Annuaire 1998, p. 245). 
Ce modèle est remplacé par un système dual de recrutement. Les autorités relèvent que comme le deuxième cercle ne revêt qu'une importance mineure pour le recrutement des travailleurs étrangers, l'introduction du système dual n'entrâ̂nera dans la pratique aucun changement important pour l'économie. La commission Hug proposait plutôt de mettre en application un système de points obtenus en fonction des qualifications professionnelles et linguistiques des candidats non européens à l'immigration. Le Conseil fédéral se garde d'y souscrire sans autre: il faudra approfondir cette nouvelle porte d'admission avant de la mettre éventuellement en application.

Dans cette même ordonnance, le Conseil fédéral propose de réduire le nombre de permis saisonniers pour la période 1998-1999, qui passeraient de 99'000 à $88^{\prime} 000$. Une telle disposition vise deux objectifs: tenir compte de la crise économique dans les secteurs d'emploi des saisonniers et se préparer à la suppression du statut du saisonnier prévue par les négociations bilatérales.

Par ailleurs, en Suisse aussi, se pose le problème des sans-papiers: dans le cadre du $150^{\mathrm{e}}$ anniversaire de la Confédération et du $50^{\mathrm{e}}$ anniversaire de la Déclaration universelle des droits de l'homme, 112 conseillers nationaux, de presque tous les courants, ont demandé, fin décembre 1997, une amnistie pour les sans-papiers vivant en Suisse ${ }^{9}$. On espère que cette motion sera traitée dans la session de décembre 1998. L'exécutif suggère la transformation de la motion en un postulat, qui est moins contraignant.

\section{$\square$ Politique d'intégration}

Suivant les recommandations de la Commission Hug, le Conseil fédéral va concentrer son effort législatif sur la révision totale de l'actuelle Loi sur le séjour et l'établissement des étrangers (LSEE) dont les travaux ont déjà commencé à l'intérieur de l'administration. Tout en maintenant ce cap pour début 1999, il a cependant lancé une révision partielle dans l'arène du Parlement dans le but d'ajouter l'intégration aux objectifs traditionnels de la politique à l'égard des étrangers, à savoir un rapport équilibré entre populations autochtone et étrangère et une saine politique du marché du travail. Ce faisant, il prend acte du rapport ${ }^{10}$ publié en juin 1996 par la Commission fédérale des étrangers (CFE) ${ }^{11}$ qui demandait à la Confédération d'assumer une partie des responsabilités en cette matière, largement laissées auparavant à la bonne volonté individuelle, à la capacité d'organisation des communautés étrangères ainsi qu'aux communes et aux cantons.

Le 10 juin 1998, à la troisième tentative, les Chambres fédérales ont donné une base légale à la politique fédérale d'intégration en votant l'article 25 de la LSEE. On attend pour la fin de cette même année un rapport de la CFE qui, synthétisant la procédure de consultation autour du texte de 1996, servira de préparation pour l'ordonnance sur l'intégration. L'article de loi doit entrer en vigueur au $1^{\text {er }}$ juillet 1999; il permettra à la Confédération de cofinancer à titre subsidiaire des projets bénéficiant déjà du financement d'une commune, d'un canton ou de tiers, qu'ils soient des communautés immigrées, des associations humanitaires ou des ser-

9. La France et l'Italie se sont déjà engagées sur cette voie et la Grande-Bretagne s'apprête à le faire; $c f$. «Janusköpfige Reform der britischen Asylpolitik», Neue Zürcher Zeitung, 31.7.98.

10. Commission fédérale des étrangers, Esquisse pour un concept d'intégration, CFE, Berne, 1996.

11. Fondée en 1970, la CFE, organe consultatif, demeure à ce jour la seule institution sur le plan fédéral dont la tâche est celle de favoriser l'intégration des étrangers. 
vices d'aide aux étrangers. Un montant de 15 millions de francs a été articulé, mais le budget sera arrêté au printemps prochain.

A la mi-octobre 98, 650 associations d'étrangers ont remis une pétition au Conseil fédéral ${ }^{12}$ demandant que la mise en pratique de la politique d'intégration, qui touche aux domaines de l'éducation, de la santé, de la culture et des affaires sociales, soit confiée au Département de l'intérieur et non pas à l'Office des étrangers (nouvelle dénomination de la Police des étrangers, responsable depuis 1931 de la mise en œuvre de la politique à l'égard des étrangers), qui relève du Département de justice et police.

Le fait que l'on approche d'un heureux dénouement ne doit pourtant pas faire oublier que le dossier politique d'intégration progresse très lentement, puisque la première impulsion date de 1990; face aux lenteurs fédérales, l'initiative dans le domaine de la politique d'intégration est passée à des acteurs situés à l'échelon subnational, notamment à certains cantons et communes, selon une sorte de principe de subsidiarité.

Les régions les plus touchées par l'immigration, notamment les centres urbains, se sont mobilisées à cet effet: en 1996 déjà, le canton de Neuchâtel s'est doté de la première loi sur l'intégration des étrangers; la même année, la Ville de Berne a publié son Leitbild sur la politique d'intégration, dont l'élaboration avait été confiée à l'Institut d'ethnologie; les deux Bâles, Lucerne et le Tessin sont en train de mettre au point leur propre politique. De son côté, Lausanne a inauguré en mars 1998 sa Chambre cantonale consultative des immigrés, calquée sur le modèle existant depuis plusieurs années au niveau de la Ville de Lausanne, alors que Genève, après avoir lancé une Fondation pour l'intégration des immigrés, remet sur le métier la question des droits civiques, le gouvernement ayant déposé un projet de loi visant à permettre aux communes qui le désirent d'octroyer le droit de vote aux étrangers porteurs d'un permis $\mathrm{C}$.

Mais les débats les plus vifs autour des initiatives locales en faveur de l'intégration se sont sans conteste déroulés en ville de Zurich, autour de deux événements: en mai, la Ville a publié un Leitbild sur l'intégration plutôt controversé et, en juin, un référendum de l'UDC contre un projet de soutien aux familles kosovares a abouti au refus d'une initiative par ailleurs très modeste. Selon certains observateurs ${ }^{13}$, l'ombre de l'UDC plane sur le modèle de Zurich: celui-ci traite les étrangers presque exclusivement comme groupe à problèmes, ne prend guère en considération le point de vue des étrangers et veut freiner l'arrivée de nouveaux immigrants. Par contre, le document bernois fait figure de pionnier: il est considéré plutôt comme un modèle parce que plus libéral là où il plaide en faveur d'une naturalisation facilitée et d'une plus grande participation des étrangers à la vie publique. L'âpre critique dont le modèle zurichois a fait l'objet, notamment dans les communautés immigrées ${ }^{14}$, a poussé les autorités à une certaine prudence dans la mise en œuvre des recommandations ${ }^{15}$.

12. «Integration gegen uns? Ohne uns!», Tages Anzeiger, 20.10.98.

13. «Pflichtaufgabe Integration», Tages Anzeiger, 25.7.98.

14. «Les étrangers ne doivent pas être considérés comme un problème», Le Temps, 27.7.98.

15. «Stadtpräsident Estermann verteidigt das Integrationsleitbild nur halbherzig», Die Wochenzeitung, 27.8.98. 


\section{SOURCES}

Asylon, revue de l'Office fédéral des réfugiés, parutions en 1988.

Asyl, Schweizerische Zeitschrift für Asylrecht und Praxis, Schweizerische Flüchltingshilfe, Bern.

Chronologie der Flüchtlingspolitik, Schweizerische Flüchtlingshilfe, parutions en 1998.

Vivre ensemble, bulletin de liaison pour la défense du droit d'asile, Genève.

Office fédéral des réfugiés, Statistique en matière d'asile 1997, ODR, janvier 1998.

Office fédéral des réfugiés, Communiqués de presse, parutions en 1998.

Der Bund, «Ausschaffung/Mehrere Kantone weigern sich, den Direktiven des Bundes zu folgen und bosnischen Flüchtlinge in ihre Heimat zurückzuschaffen. Sie werden jedoch bestraft», 10.8.98

Carrefour, «Loi sur l'intégration des étrangers. Que va-t-il se passer maintenant?», 26.9.98.

Le Courrier, «Algérie. Appel du HCR», 22.1.98; «Miroir aux alouettes pour les femmes», 7.4.98; «J-3. Le canton de Vaud a mené une résistance opiniâtre», 28.4.98; «600 enseignants demandent un sursis au renvoi des enfants», 22.7.98; «De grâce, amnistiez les sans-papiers!», 4.10.98.

L'Hebdo, «Bosniaques: le couperet tombe. Expulsions: la Confédération reste ferme sur les renvois, mais certains cantons résistent», 28.9.97; «Mieux vaut être requérant à Nidwald qu'en Valais», 23.7.98.

Journal de Genève, «Pour Jean-Daniel Gerber, l'Office des réfugiés n'est pas qu'une machine à expulser les requérants d'asile», 12.12.97; «Algérie: Berne aux abonnés absents», 7.1.98; «La Suisse devrait revoir les conditions de rapatriement des Bosniaques», 31.1.98

Neue Zürcher Zeitung, «Probleme im Asylbereich», 1.3.98; «Abschied vom Drei-Kreise-Modell», 9.6.98; «Kantonale Diskriminierung von Asylbewerbern? Evaluation zur Praxis der Flücklingsanerkennung», 6.7.98; «Asylpolitischer Echtfall», 21.10.98; «Evaluation des Rückkehrhilfeprogramms: Gemischte Bilanz in Bosnien-Herzegowina», 31.10.98.

Le Nouveau Quotidien, «Berne ne veut rien changer à la politique de rapatriement des réfugiés algériens», 8.1.98; «La Suisse froide devant les massacres», 8.1.98; «Hantés par la tragédie algérienne, des Suisses commencent à se mobiliser", 21.1.98.

Tages Anzeiger; «Damit streut man dem Volk Sand in die Augen», 7.7.98; «Pflichtaufgabe Integration», 25.7.98; «In 2 Tagen laüft die Frist ab - und dann?», 29.7.98; «Integration gegen uns? Ohne uns!», 20.10.98; «Koller: «Keine kollektive Aufnahme»», 21.10.98.

Le Temps, «La situation ayant changé en Allemagne, la Suisse suspend le renvoi des Kosovars», 12.6.98; «Asile, la situation devient chaude», 17.6.1998; «L'USS soutient le double référendum contre l'asile sans se faire d'illusions», 10.7.98; «L'Espace européen de coopération policière s'entrouvre à la Suisse», 17.7.98; «Les étrangers ne doivent pas être considérés comme un problème», 27.7.98; «Le sort des Bosniaques en Suisse se joue sur la marge de manœuvre des cantons. Tour d'horizon des frondes cantonales», 8.8.98; «Le retour vers la précarité», 8.9.98.

Tribune de Genève, «Nous n'avons pas à rougir de notre politique d'asile», 28.1.98; «Laissez les jeunes Bosniaques terminer leur formation», 29.7.98; «Le Conseil fédéral choisi l'urgence pour verrouiller sa politique d'asile», 12.3 .98

Die Wochenzeitung, «Stadtpräsident Estermann verteidigt das Integrationsleitbild nur halbherzig», 27.8.98.

\section{SITES INTERNET}

Haut-Commissariat des Nations Unies pour les Réfugiés: http://www.unhcr.ch

Journal Le Courrier: http://www.imaginer.ch/courrier/réfugiés

Comité référendaire contre le démantèlement du droit d'asile: http://www.asile.ch; e-mail:asile@worldcom.ch

\subsection{CORRUPTION - BLANCHIMENT D'ARGENT - FUITE DE CAPITAUX}

On sait que la Suisse garantit dans une large mesure le secret bancaire aux personnes physiques et morales qui déposent dans une banque suisse de l'argent acquis légalement. Mais, selon Peter F. Mueller, qui a étudié de près le secret bancaire suisse ${ }^{16}$, cette idée "ne peut être jugée positive qu'à condition de reconnaître en même temps les risques qui y sont liés et les effets négatifs éventuels, de les enregistrer objectivement et de leur appliquer des sanctions efficaces sans concessions en cas d'abus [...] Dans la majorité des pays, notamment dans les grandes nations industrielles, en premier lieu aux Etats-Unis, cette conception généreuse de la protection des droits de la personne, qui couvre aussi sa fortune, n'est généralement pas partagée par le législateur. Dans ces pays, lorsqu'il s'agit par exemple d'avoir accès à des fonds pour prélever directement des impôts, les intérêts de l'Etat priment ceux de la sphère privée des personnes. Malgré les conflits, il est urgent de rapprocher autant que possible, dans leur intérêt, les règlements en vigueur en Suisse de ceux d'autres Etats».

16. Peter F. Mueller, Wegleitung zum schweizerischen Bankgeheimnis. Grundlagen, Rechtshilfe, Ausblick, Schulthess, Zürich, 1998 
Quelles que soient les réserves de fond que l'on puisse avoir sur ces conceptions suisses et leur application, la présentation de Peter F. Mueller montre clairement deux choses. En premier lieu, la Suisse a une responsabilité particulière, en raison de sa réglementation, en matière de lutte contre la corruption, le blanchiment d'argent et la fuite de capitaux. Nous présentons ci-après les efforts réalisés et les problèmes rencontrés. En deuxième lieu, le fait qu'en Suisse l'évasion fiscale ne soit pas punissable, à la différence de la fraude fiscale, lui a valu ces dernières années de plus en plus de critiques sur la scène internationale, notamment de la part de l'OCDE. La coopération internationale, dans ce domaine si lucratif pour la place financière suisse, pourrait bien être le principal défi auquel le pays est confronté aujourd'hui.

\subsubsection{CORRUPTION}

La question de la corruption a occupé ces dernières années une grande place dans les débats publics. Des cas patents de dessous-de-table - l'affaire de l'ex-fonctionnaire zurichois Raphael Huber ou celle du colonel Friedrich Nyffenegger par exemple -, mais aussi une série d'autres scandales qui se sont produits dans des cantons ou des communes ont fait que la corruption n'est plus un sujet tabou en Suisse. Ces exemples ont aussi mis en évidence les limites des articles du Code pénal punissant la corruption, qui datent pour l'essentiel de 1942. Les pots-devin dans les transactions commerciales internationales représentent en même temps un grave problème actuel. Ils n'entraînent pas seulement des distorsions de la concurrence, mais compromettent aussi l'efficacité des mesures de politique du développement. Partout où règne la corruption, elle est une menace pour l'Etat de droit et la démocratie.

La lutte contre la corruption internationale réclame une mise en commun des efforts; c'est pourquoi l'OCDE a mis au point des directives internationales, aboutissement d'une élaboration de plusieurs années à laquelle la Suisse a participé activement. La Convention de l'OCDE sur la lutte contre la corruption d'agents publics étrangers dans les transactions commerciales internationales ${ }^{17}$ a été signée en décembre 1997 par les représentants de 33 pays; la Suisse était représentée par le conseiller fédéral Jean-Pascal Delamuraz.

G ASTM 1998, pp. 252-253, «Convention de l'OCDE contre la corruption».

$\square$ Révision du droit pénal suisse

En signant la convention de l'OCDE, la Suisse s'engageait à adapter ses lois en 1998, pour qu'elles tiennent compte de ses recommandations. Contrairement à plusieurs autres pays, elle n'a pas su respecter ce délai. Il existe toutefois un rapport explicatif avec un avant-projet concernant la révision du droit pénal suisse sur la corruption ${ }^{18}$, que le Conseil fédéral a envoyé en consultation le $1^{\text {er }}$ juillet 1998. Fondé essentiellement sur un avis de droit établi par le professeur bâlois Mark Pieth, en collaboration avec Marco Balmelli, docteur en droit, cet avantprojet comporte trois volets, consacrés l'un à la corruption des agents publics

17. Le texte de la convention ainsi que de vastes informations sur son application dans les différents pays peuvent être consultés sur le site Internet de l'OCDE: http://www.oecd.org/daf

18. Révision du droit pénal suisse sur la corruption. Rapport et avant-projet, Berne, juin 1998. On peut notamment l'obtenir auprès de l'OFAEE. 
suisses, un autre à celle des agents publics étrangers et le troisième à la corruption privée ${ }^{19}$. Voici leurs éléments principaux ${ }^{20}$ :

口 Il importe que les dispositions du Code pénal sur la corruption des agents publics suisses soient mieux mises en accord et réunies sous un seul titre. A l'avenir, la corruption active doit être considérée comme un crime (jusqu'ici, il s'agissait d'un délit). Cela permettra de rallonger le délai - aujourd'hui trop court - permettant de poursuivre ce genre d'infraction. En outre, le blanchiment des gains illicites que sont les pots-de-vin sera systématiquement punissable. Par ailleurs, il y aura aussi corruption lorsqu'il sera accordé ou demandé une commission occulte après la prestation d'un acte officiel. Enfin, les nouvelles normes pénales sur l'octroi ou l'acceptation d'un avantage personnel ne s'étendront pas seulement aux cadeaux consentis pour un acte légal isolé, mais aussi aux largesses accordées pour la simple gestion des intérêts publics. Cela permettra de punir les méthodes typiques de mise en place de réseaux particulièrement dangereux de corruption systématique basée sur l'«alimentation progressive» ou l' «entretien d'un climat propice».

- La nouvelle disposition relative à la corruption d'agents publics étrangers comble une grosse lacune dans les moyens à disposition pour combattre la corruption internationale. Elle remplit en outre la condition première qui est exigée pour la ratification de la convention de 1'OCDE sur la lutte contre la corruption de décembre 1997.

口 Le droit en vigueur réprime déjà la corruption privée. Pourtant, les dispositions s'y rapportant dans la Loi fédérale contre la concurrence déloyale (LCD) sont quasiment restées sans effet. La révision entend y remédier en rendant punissables non seulement la corruption privée active, mais aussi la corruption passive. Par ailleurs, ces infractions ne seront plus poursuivies sur plainte, mais d'office.

Les deux premiers volets de l'avant-projet de loi ont rencontré une large approbation dans la procédure de consultation. Par contre, la formulation du troisième volet relatif à la révision du droit en matière de corruption privée a provoqué une forte opposition. C'est pourquoi le Conseil fédéral a décidé de séparer cette partie de 1'avant-projet et de la présenter ultérieurement, dans un deuxième paquet portant sur la ratification de la convention du Conseil de l'Europe sur les mesures pénales contre la corruption. Pour la révision des dispositions pénales relatives à la corruption d'agents publics, le Département fédéral de justice et police (DFJP) élaborera ces prochains mois un message, au sujet duquel le Conseil national et le Conseil des Etats prendront position au plus tôt à la session d'automne 1999, au même moment où ils auront à ratifier la convention de l'OCDE.

\section{$\square$ Mesures préventives contre la corruption}

Le droit pénal à lui seul n'est pas à même d'empêcher la corruption. C'est pourquoi les directives de l'OCDE pour la lutte contre la corruption internationale prévoient trois mesures préventives supplémentaires ${ }^{21}$. Complétées par une quatrième mesure, elles devraient être appliquées en Suisse.

19. La proposition dans ce domaine va au-delà des directives de l'OCDE, qui se limitent actuellement au secteur public Mais l'OCDE envisage aussi de les élargir.

20. Communiqué de presse du Département fédéral de justice et police, 1.7.98.

21. OFAEE, Mesures de l'OCDE pour la lutte contre la corruption internationale, juillet 1998. 


\section{Suppression de la déductibilité fiscale des pots-de-vin}

Selon la loi actuelle, les dessous-de-table peuvent toujours être déduits des déclarations d'impôts s'ils peuvent être considérés comme des frais d'acquisition. Il faut pouvoir donner la preuve que certains revenus n'ont pu être obtenus que grâce à ces paiements. Suite à l'initiative parlementaire du conseiller national Werner Carobbio, la Commission de l'économie et des redevances du Conseil national a préparé une proposition de modification de la Loi fédérale sur l'impôt fédéral direct et de la Loi fédérale sur l'harmonisation des impôts directs des cantons et communes, modification visant à interdire la déductibilité fiscale des pots-de-vin. Cette proposition a été approuvée par le Conseil fédéral, qui l'a donnée en consultation aux cantons. Au moment où nous mettons sous presse, les nouveaux projets de loi ne sont pas encore disponibles.

[D ASTM 1998, pp. 253-254, «Le Conseil fédéral veut supprimer la déductibilité fiscale des pots-de-vin».

\section{Accroissement de la transparence comptable}

L'introduction de prescriptions en matière comptable (comme l'interdiction de tenir des comptes en parallèle à la comptabilité de l'entreprise) et le renforcement des contrôles effectués par des vérificateurs externes et internes doivent permettre de découvrir le versement de pots-de-vin, voire de l'empêcher. Les principes généraux - fixés par le Code des obligations - de l'établissement des rapports financiers satisfont largement, dans leur esprit, la recommandation de l'OCDE. Une consultation aura encore lieu cette année sur le projet d'une loi fédérale sur l'établissement et la vérification des comptes et du bilan, qui doit être conforme au droit européen.

\section{Accroissement de la transparence des acquisitions publiques}

L'essentiel des vœux contenus dans cette recommandation est déjà inscrit dans la loi suisse.

\section{Réglementation des cadeaux reçus par les agents publics}

Il n'est pas toujours aisé de tracer une limite entre cadeaux légitimes et dessousde-table; c'est pourquoi des règles claires sont nécessaires. Le Département fédéral des finances est en train d'élaborer un projet à l'intention de la Confédération.

\section{$\square$ Activités diverses de lutte contre la corruption en Suisse}

En 1998, l'actualité et l'urgence du problème de la corruption en ont fait parler à plusieurs reprises au niveau fédéral; le rapport annuel sur les affaires économiques extérieures du Conseil fédéral fait notamment allusion à la lutte contre la corruption. La commission consultative du Conseil fédéral pour la coopération et le développement a consacré à ce thème une de ses réunions à huis clos, et la Direction du développement et de la coopération (DDC) étudie l'insertion de clauses sur la corruption dans ses contrats. Dans le cadre d'un projet au Cameroun cofinancé par la DDC et Transparency International, Pain pour le prochain examine le rôle des organisations non gouvernementales dans la lutte contre la corruption.

\section{$\square$ L'engagement de la Banque asiatique}

de développement et de la Banque mondiale contre la corruption

La crise économique qui a éclaté en Asie a provoqué l'ouverture d'enquêtes officielles contre des structures économiques corrompues. La Banque asiatique de développement (BAsD) a publié un nouveau texte de stratégie contre la corrup- 
tion, donnant ainsi priorité aux mesures à prendre sur cette question ${ }^{22}$. On a en effet découvert que dans plusieurs pays d'Asie l'Etat paie des biens et des services à des prix qui sont surévalués de 20 à $100 \%$ en raison de la corruption, ce qui peut leur coûter jusqu'à la moitié de leurs recettes fiscales. Dans certains pays, le montant des pertes subies depuis des décennies par des pratiques de corruption est plus important que celui de la dette extérieure. La nouvelle politique anticorruption mise en œuvre par la BAsD cherche entre autres à consolider les institutions publiques en exigeant un contrôle renforcé de leurs structures et une meilleure transparence. Elle soutient les pays qui entreprennent de lutter contre la corruption. Cette question sera aussi intégrée à l'avenir dans les négociations entre la BAsD et les gouvernements.

Depuis la fin de 1994, la Banque mondiale (BM) a elle aussi introduit des programmes de lutte contre la corruption dans sa stratégie de good governance. A la différence de l'OCDE, elle ne s'occupe que de corruption passive, tant dans le cadre de ses propres projets que dans l'encouragement aux Etats demandeurs à édicter des réglementations à ce sujet ${ }^{23}$.

\section{$\square$ Avis critiques}

Divers avis expriment des inquiétudes à l'encontre des efforts réalisés par l'OCDE et d'autres institutions internationales pour lutter contre la corruption. Dani Rodrik, professeur d'économie internationale à l'Université de Harvard ${ }^{24}$, constate notamment que ce sont les limitations de la concurrence, résultant de paiements illégaux, entre des entreprises ayant pour la plupart leur siège dans des pays industrialisés qui ont mis cette question sur le tapis. Les mesures anticorruption, dont il ne nie pas l'utilité, ne doivent pas faire oublier que ceux qui les ont instaurées s'intéressent avant tout à gagner des parts du marché, plus qu'ils ne se soucient sérieusement du bien public. Dani Rodrik craint que le problème national de la corruption, qui dans nombre de pays affecte le développement et l'économie, ne se confonde avec les problèmes que la corruption entraîne pour l'économie internationale. Il s'agit en effet de deux choses différentes, même si les efforts actuels pour lutter contre la corruption peuvent en partie être utiles sur les deux plans. Cette question réclame une plus grande attention à l'avenir.

\subsubsection{BLANCHIMENT D'ARGENT}

\section{Loi sur le blanchiment d'argent}

La Loi fédérale concernant la lutte contre le blanchiment d'argent dans le secteur financier (LBA) ${ }^{25}$ est entrée en vigueur le $1^{\mathrm{er}}$ avril 1998. Elle comporte deux nouveautés importantes. En premier lieu, elle s'applique aux intermédiaires financiers, soit à toutes les personnes qui, à titre professionnel, acceptent, gardent en dépôt ou aident à placer ou à transférer des valeurs patrimoniales appartenant à des tiers. L'obligation de diligence consistant notamment à vérifier l'identité du cocontractant et de l'ayant droit économique ne touche donc plus seulement les

22. Financial Times, 9.7.98.

23. Weltbank, Informationsblätter Dritte Welt, Oktober 1998.

24. Finanzplatz Informationen, 1/98.

25. «Loi fédérale concernant la lutte contre le blanchiment d'argent dans le secteur financier (Loi sur le blanchiment d'argent, LBA)», Feuille fédérale, n ${ }^{\circ} 41$, vol. $4,21.10 .97$, pp. 723 et suiv. 
banques et les assurances, mais aussi tous les intermédiaires non soumis au contrôle bancaire tels que les fiduciaires, notaires, gérants de fortune et avocats. Ils sont tenus de clarifier le but de toute transaction qui laisse planer un doute et de conserver les pièces à ce sujet.

En second lieu, il n'y a plus droit mais devoir d'informer. Désormais, tous les intermédiaires financiers doivent signaler au Bureau de communication en matière de blanchiment d'argent, créé en février 1998, toute information et tout soupçon fondé de blanchiment. Les valeurs concernées seront bloquées au maximum durant cinq jours sans que le client en soit informé. Pendant ce temps, les collaborateurs du Bureau de communication poursuivent les recherches. Si le soupçon de blanchiment se confirme, le cas est dénoncé à l'autorité de poursuite pénale compétente. Dans le cas contraire, le blocage des avoirs est levé.

Une autorité de contrôle, rattachée à l'Administration fédérale des finances, veille à ce que les intermédiaires financiers respectent leurs obligations. Comme la Loi sur le blanchiment d'argent repose sur le principe de l'autorégulation, un délai de deux ans leur a été accordé pour choisir le type de contrôle qu'ils souhaitent, en s'affiliant soit directement au Bureau de communication à Berne, soit à l'organisme d'autorégulation de leur branche professionnelle. Ces organismes, par analogie avec l'Association suisse des banquiers, doivent veiller à ce que les banques observent les obligations que leur fait la Loi sur le blanchiment d'argent et imposer des sanctions en cas d'infraction. A la fin novembre, l'Administration fédérale des finances avait reçu de six associations la demande d'être reconnues comme organismes d'autorégulation. Les décisions en la matière seront prises d'ici au mois d'avril 1999.

@U ASTM 1998, pp. 248-250, «Loi sur le blanchiment d'argent dans le secteur financier».

\section{$\square$ Premier bilan du Bureau de communication en matière de blanchiment d'argent}

A la fin octobre, 96 transactions suspectes d'un montant total de 275 millions de francs avaient été communiquées au Bureau de communication; 61 d'entre elles ont été transmises à l'autorité pénale ${ }^{26}$. On voit là que depuis l'entrée en vigueur de la nouvelle loi, les annonces connaissent une nette croissance: ces dernières années, les autorités pénales avaient en effet à connaître 30 à 40 cas par an. Seuls $20 \%$ des cas enregistrés depuis le mois d'avril ont été annoncés par des intermédiaires financiers. (Pour les motifs, voir le paragraphe suivant.) Pour ce qui est des banques, on ne constate presque plus aucune résistance contre l'obligation d'informer. Cela ne change toutefois rien au fait que la politique d'information des banques reste assez restrictive.

\section{$\square$ Activités du Groupe d'action financière de l'OCDE}

\section{Rapport sur la Suisse}

Le rapport du Groupe d'action financière sur le blanchiment d'argent de capitaux (GAFI) pour 1997-1998 ${ }^{27}$ examine les bases de la lutte contre le blanchiment d'argent en Suisse. Le GAFI salue les innovations apportées par la nouvelle Loi sur le blanchiment d'argent. Il déplore par contre l'absence de contrôle - bien que celui-ci soit prévu - sur le respect de l'obligation de diligence par les inter-

26. Bureau de communication en matière de blanchiment d'argent, 2.11.98.

27. GAFI, Rapport annuel 1997-1998, juin 1998. Disponible sur Internet: http://www.oecd.org/fatf/. Rapport sur la situation légale en Suisse, pp. 14-15. 
médiaires financiers et constate l'importance du rôle des autorités de surveillance pour convaincre les acteurs financiers de la nécessité de participer à la lutte contre le blanchiment d'argent. Le GAFI critique en particulier l'absence de réelles compétences de poursuite au niveau fédéral; on sait qu'en Suisse elles sont du ressort des pouvoirs judiciaires cantonaux.

\section{Nouvelles formes de blanchiment d'argent}

L'essentiel du rapport annuel du GAFI pour 1997-1998 porte sur la présentation et la classification des principales méthodes de blanchiment d'argent. On constate que leurs formes évoluent. L'utilisation de nouvelles technologies - transferts d'argent via Internet, cartes à puce et porte-monnaie électroniques - devient de plus en plus fréquente et représente un nouveau défi pour les possibilités de poursuites pénales. En outre, avec le renforcement des réglementations dans le secteur financier, le blanchiment d'argent s'est partiellement transféré au négoce d'œuvres d'art, de produits de luxe et de propriétés immobilières. Les experts montrent une certaine inquiétude face à l'introduction de la nouvelle monnaie européenne, car celle-ci risque de permettre à ceux qui blanchissent de l'argent de brouiller les pistes. De nouvelles tâches sont à prévoir, mais jusqu'ici ni le GAFI ni les autorités suisses ne se sont encore penchés concrètement sur la question.

C] ASTM 1998, p. 250, «Activité du Groupe d'action financière de l'OCDE (GAFI)».

\section{$\square$ Loi fédérale sur les jeux de hasard et les maisons de jeu}

(Loi sur les maisons de jeu, LMJ) ${ }^{28}$

Le 7 mars 1993, l'initiative constitutionnelle sur la légalisation des jeux de hasard était adoptée en votation populaire. Les Chambres ont discuté de la loi fédérale lors de leur session de septembre 1998 et l'ont mise au point à la session de décembre. Tout comme le secteur parabancaire, les maisons de jeu (avec ou sans tables de jeu) seront soumises à la Loi fédérale sur le blanchiment d'argent dans le secteur financier. Au sens de l'article 2, alinéa 2, de la loi sur le blanchiment d'argent, les maisons de jeu sont en effet réputées «intermédiaires financiers».

Les maisons de jeu peuvent être des terrains propices au blanchiment d'argent sous deux aspects. D'une part, elles ont un chiffre d'affaires élevé et revêtent donc un intérêt pour le blanchiment d'argent au même titre que les hôtels, le tourisme, les centres commerciaux et les restaurants. Elles peuvent représenter un investissement intéressant et servir à leurs gérants ou à des cadres corrompus d'outil pour le blanchiment d'argent. D'autre part, les usagers peuvent se livrer à un emploi abusif des maisons de jeu pour blanchir leur argent. La Loi sur les maisons de jeu non seulement soumet les maisons de jeu à la Loi sur le blanchiment d'argent, mais elle prévoit en outre plusieurs mesures pour les préserver du blanchiment d'argent (les ordonnances détaillées doivent encore être promulguées):

๖ Lors d'une demande de concession, le requérant doit présenter un programme de mesures de sécurité qui définit les mesures prévues pour lutter contre la criminalité et le blanchiment d'argent. L'autorité de surveillance (la Commission fédérale des maisons de jeu) veille à ce que les conditions soient respectées. Si tel n'est pas le cas, elle peut prendre des sanctions et notamment suspendre la concession.

28. «Loi fédérale sur les jeux de hasard et les maisons de jeu (Loi sur les maisons de jeu, LMJ)», projet du Conseil fédéral du 26 février 1997, loi fédérale, Feuille fédérale, nº51, 30.12.98, pp. 5004 et suiv. 
- Pour qu'une concession soit délivrée, le requérant doit établir l'origine des fonds investis et de leurs partenaires. Cette exigence de transparence vaut aussi après l'octroi de la concession. La preuve de l'origine licite vaut non seulement pour les mises de fonds propres, mais aussi pour celles de capitaux étrangers. La demande de concession est en outre publiée dans la Feuille fédérale et dans la feuille des avis officiels du canton.

- L'autorité de surveillance est constituée par la Commission fédérale des maisons de jeu, qui jouit, comme on l'a vu, de compétences étendues. L'efficacité de la législation sur les maisons de jeu tient pour une large part à la composition de la commission: ses membres doivent être indépendants et ont obligation de publier leur rapport. La loi définit cette obligation de manière très succincte. La Commission des maisons de jeu fera partie des autorités les plus soumises à des tentatives d'influence, dans la mesure où les sommes en jeu seront importantes.

\section{$\square$ Répartition et utilisation de l'argent de la drogue placé sous séquestre}

La plus grosse affaire de blanchiment d'argent connue en Suisse, le cas de la trafiquante colombienne Sheila Arana de Nasser, et la répartition subséquente des 240 millions de francs qui avaient alors été confisqués ont montré que la base légale n'est pas satisfaisante pour des cas de ce genre. Les fonds bloqués sur un compte auprès de l'UBS ont été répartis en parts égales entre les deux Etats plaignants. Mais déjà avant que la Suisse n'ait reçu, en décembre 1998, la part qui lui revenait, une longue dispute s'est déroulée entre les cantons de Vaud et de Zurich et la Confédération. En septembre 1998, une solution a enfin pu être trouvée: les deux cantons touchent chacun $40 \%$, la Confédération $20 \%$ de la moitié due à la Suisse. Toutefois, selon le Département fédéral de justice et police, ce règlement ne doit pas constituer un précédent; pour éclaircir l'affaire, une commission d'experts a donc été nommée. Elle a pour tâche de créer une base légale pour de pareils cas et d'examiner la possibilité d'attribuer les sommes en jeu à des fins spécifiques. Dans la plupart des cantons, l'argent de la drogue est allé jusqu'ici directement dans le budget cantonal, sans que l'on se pose la question éthique et politique de l'origine délictueuse de ces fonds ou de l'indemnisation de victimes éventuelles. Font exception les cantons de Fribourg et de Genève, où des réglementations claires permettent de financer par cet argent le développement de la prévention, de la thérapie et de la lutte contre les toxicomanies ${ }^{29}$. Le Groupe de travail Suisse-Colombie s'est associé à la Communauté de travail des œuvres d'entraide pour réclamer que la commission d'experts débatte prioritairement de la question de l'attribution de ces fonds et qu'elle soit incluse dans la loi. Conformément à leurs objectifs de développement, ces deux associations proposent que l'argent de la drogue serve pour moitié à la prévention contre les toxicomanies en Suisse et pour moitié à aider les pays producteurs du tiers monde à diminuer leurs cultures.

\section{$\square$ Le cas Salinas}

Le Ministère public de la Confédération avait ouvert, en novembre 1995, une enquête de police judiciaire contre Raul Salinas de Gortari, frère de l'ancien chef de l'Etat du Mexique, son épouse Patricia Paulina Castañon de Salinas, le frère 
de celle-ci et d'autres personnes encore, toutes soupçonnées d'infractions à la loi sur les stupéfiants et de blanchiment d'argent. La lourde procédure a été close en octobre 1998. L'abondance des indices et des preuves permet d'établir que les fonds, d'un total de 114,4 millions de dollars, découverts et bloqués en Suisse proviennent du trafic de drogue et doivent être confisqués au profit de l'Etat. Il appartient aux autorités politiques de décider d'une répartition éventuelle des fonds saisis entre la Suisse et des Etats tiers d'une part, entre la Confédération et les cantons concernés d'autre part ${ }^{30}$.

\subsubsection{FUITE DE CAPITAUX ET ENTRAIDE JUDICIAIRE INTERNATIONALE}

La Loi sur l'entraide judiciaire, base de l'entraide pénale internationale, n'a pas été modifiée en 1998. Mais le Conseil fédéral a conclu avec le Pérou et l'Equateur deux nouveaux traités d'entraide judiciaire, qui doivent encore être ratifiés par les Chambres. Jusqu'ici, l'entraide judiciaire entre la Suisse et ces deux pays reposait exclusivement sur les lois nationales. Les nouveaux traités doivent simplifier et accélérer le processus d'entraide judiciaire. Selon le Département fédéral de justice et police, cela ouvre une nouvelle ère de coopération entre la Suisse et l'Amérique latine en matière d'entraide pénale internationale: il s'agit en effet là des premiers traités que la Suisse conclut avec des Etats de ce continent ${ }^{31}$.

\section{Philippines}

Après plus de douze ans, les avoirs gelés sur des comptes suisses de l'ancien dictateur Ferdinand Marcos, qui se montent à 534 millions de dollars, ont été transférés en 1998 sur un compte bloqué auprès de la Banque nationale des Philippines. Cela a été rendu possible grâce aux décisions du Tribunal fédéral de décembre 1997 et janvier 1998 donnant le feu vert à ce transfert, et au rejet des ultimes recours en avril 1998. Au-delà du cas Marcos, cette décision prend une grande importance dans la mesure où la cour suprême suisse ne s'est explicitement pas fondée seulement sur la Loi sur l'entraide judiciaire, mais aussi sur les engagements qui résultent de la signature par les deux pays de conventions internationales (en particulier la Déclaration universelle des droits de l'homme et la Convention des Nations Unies contre la torture). Le Tribunal fédéral a posé deux conditions au transfert des fonds. En premier lieu, les Philippines s'engagent à dédommager les victimes de violations des droits de l'homme sous le régime Marcos. En outre, elles doivent donner à la Suisse l'assurance que la confiscation ou la restitution de l'argent aux ayants droit se feront selon une procédure juridique conforme aux règles de procédure du Pacte international relatif aux droits civils et politiques. Les Philippines s'engagent enfin à renseigner les autorités suisses sur la procédure judiciaire de confiscation et de restitution des avoirs et sur les mesures prises pour dédommager les victimes de la torture ${ }^{32}$. Avec le transfert de l'argent effectué en avril, le cas Marcos est donc clos pour les banques suisses, mais non pas pour les autorités. Elles ont en effet la tâche de suivre d'un œil critique le respect des conditions écrites acceptés par le gouvernement des Philippines. En cas de manquement, le gouvernement suisse devrait déposer plainte auprès de la commission des Nations Unies compétente.

미 ASTM 1998, p. 251.

30. Communiqué de presse du Ministère public de la Confédération et de l'Office fédéral de la police, 20.10.98.

31. Communiqué de presse du Département fédéral de justice et police, 8.4.98.

32. Décisions du Tribunal fédéral du 10 décembre 1997 et de janvier 1998. 


\section{République démocratique du Congo (ex-Zaïre)}

Les avoirs en Suisse de Mobutu Sese Seko, gelés en mai 1997 après sa chute, resteront bloqués jusqu'à la fin de la procédure d'entraide judiciaire. L'Office fédéral de la police (OFP) le garantit dans deux décisions d'entrée en matière prises suite à la requête de la République démocratique du Congo. Les investigations conduites sur la base des indications congolaises relatives aux avoirs de la famille Mobutu n'ont toutefois pas permis de découvrir d'autres comptes que ceux qui avaient été bloqués. Sans autres indications, les autorités suisses ne peuvent pas légalement se livrer à de plus amples recherches (interdiction des fishing expeditions). En mai 1998, l'Office fédéral de la police a rappelé aux autorités congolaises qu'il avait besoin de leur coopération pour pouvoir accorder l'entraide judiciaire demandée. La guerre actuelle en République démocratique du Congo rend évidemment cette procédure plus difficile.

\section{Pakistan}

Sur demande des autorités pakistanaises, quelque 20 millions de francs ont été gelés l'an dernier sur des comptes appartenant à Benazir Bhutto, ancien premier ministre, et à ses proches. En juillet 1998, le juge d'instruction genevois Daniel Denant a demandé l'inculpation pour blanchiment du mari de Benazir Bhutto, Ali Zardari, puis de Benazir Bhutto elle-même ${ }^{33}$.

ASTM 1998, p. 252.

\section{Ethiopie}

En novembre 1997, l'Ethiopie avait demandé l'entraide judiciaire à la Suisse dans le cadre de la procédure pénale à l'encontre de Tamirat Layne, ancien premier ministre. Il lui est reproché ainsi qu'à ses proches d'avoir détourné des fonds de 1991 à 1995 et de les avoir déposés en partie à Genève. Le 5 décembre 1997, l'Office fédéral de la police a bloqué 8 millions de dollars déposés sur divers comptes dans une banque genevoise. A la fin mars 1998, l'OFP a ordonné que les documents bancaires soient transmis aux autorités éthiopiennes et que les fonds bloqués leur soient restitués. Une première partie a été versée le 18 septembre. En raison d'une plainte contre cette décision de l'OFP, le Tribunal fédéral a d'abord suspendu la restitution du reste des documents et a exigé de la part du gouvernement éthiopien des garanties pour un déroulement du procès conforme aux droits de l'homme. Une garantie lui a été fournie depuis lors. A la fin décembre 1998, le reste des documents bancaires a ainsi pu être transmis à l'Ethiopie. Le Tribunal fédéral met pour condition au transfert des fonds qu'un jugement définitif et exécutoire soit prononcé par les tribunaux éthiopiens ${ }^{34}$.

\section{Indonésie}

Le 21 mai 1998, le général Suharto, qui avait dirigé l'Indonésie en dictateur pendant plus de trente ans, a finalement dû se retirer sous la pression populaire. Pour se maintenir au pouvoir et préserver ses intérêts économiques, une élite peu nombreuse avait multiplié les violations des droits de l'homme en Indonésie et dans les territoires occupés. La fortune de la famille Suharto, qui contrôlait une grande partie de l'activité économique du pays par des manœuvres frauduleuses, est estimée entre 4 et plus de 40 milliards de dollars ${ }^{35}$. On suppose qu'une partie de cette

34. Communiqués de presse de l'Office fédéral de la police, 2.4.98 et 18.9.98; Tagesanzeiger, 22.12.98.

35. Pour déterminer l'ordre de grandeur de transactions financières illégales, on ne peut généralement se fonder que sur des estimations qui se contredisent très souvent. Il en est ainsi des fonds accumulés par Suharto. 
fortune a été déposée en Suisse ${ }^{36}$; c'est pourquoi diverses organisations non gouvernementales (Action Place financière Suisse, Déclaration de Berne, Pain pour le prochain) ainsi que des parlementaires fédéraux et des membres de commissions fédérales ont demandé au Conseil fédéral de bloquer préventivement les éventuels fonds de Suharto et de son entourage se trouvant en Suisse. Il n'a pas été donné satisfaction à cette requête, car le successeur de Suharto à la tête du gouvernement, son ami B.J. Habibie, n'a déposé aucune demande d'entraide judiciaire à la Suisse. On ne sait pas encore si cela se fera après les élections promises. D'ici là, il est fort probable que d'éventuels «fonds Suharto» en Suisse auront été déposés ailleurs.

\section{Argentine}

La Suisse soutient la procédure pénale ouverte par l'Espagne contre 100 militaires argentins inculpés de meurtre et de privations de liberté à l'encontre de citoyens espagnols ainsi que du crime imprescriptible de génocide, de vol et de falsification d'actes officiels à l'époque de la dictature militaire (de 1976 à 1983). Le 20 janvier 1998, l'Office fédéral de la police a fait parvenir à l'ambassade d'Espagne à Berne des documents bancaires obtenus par l'entraide judiciaire $^{37}$.

En outre, la Suisse a accordé l'entraide judiciaire à l'Argentine dans le cadre d'une affaire de pots-de-vin touchant la Banque d'Etat argentine ${ }^{38}$.

\section{Chili}

La Suisse est aussi impliquée dans la procédure en cours contre l'ancien dictateur chilien Augusto Pinochet. En octobre 1998, lors d'un séjour en Angleterre, ce dernier a été arrêté en raison de la demande d'extradition déposée par les autorités espagnoles. Les autorités judiciaires britanniques ont reconnu le bien-fondé de la demande d'extradition en décembre 1998. La procédure doit toutefois être reprise depuis le début, la récusation pour opinion préconçue d'un des pairs de justice ayant été demandée. Au moment où nous mettons sous presse, on ne sait pas encore si l'Espagne pourra poursuivre sa procédure pénale contre Pinochet pour enlèvement et assassinat de citoyens espagnols.

Après la demande de l'Espagne et suite au mandat décerné par le procureur genevois Bernard Bertossa, l'Office fédéral de la police a lui aussi demandé, le 26 octobre 1998, que Pinochet soit maintenu en détention extraditionnelle. L'instruction genevoise se fonde notamment sur l'inculpation d'assassinat du double national suisse et chilien Alexei Jaccard.

\section{Angola}

En novembre 1998, le Conseil fédéral ordonnait de geler les avoirs de Jonas Savimbi, dirigeant de l'Unita (Union nationale pour l'indépendance totale de l'Angola), et d'autres militaires de l'Unita. Cette décision ne se fondait pas sur la Loi sur l'entraide judiciaire, mais répondait à la décision de l'été 1998 des Nations Unies de prononcer des sanctions. Cette mesure ainsi que d'autres doivent pousser l'Unita à respecter l'accord de paix conclu avec le gouvernement de l'Angola. 


\section{SOURCES}

Peter F. Mueller, Wegleitung zum schweizerischen Bankgeheimnis. Grundlagen, Rechtshilfe, Ausblick, Schulthess, Zürich, 1998.

Corruption

Révision du droit pénal suisse sur la corruption. Rapport et avant-projet, Berne, juin 1998 (peut être obtenu notamment auprès de l'OFAEE).

Aktion Finanzplatz Schweiz, «Kampf gegen Schmiergelder - Globalisierung macht's möglich», Aktion Finanzplatz Schweiz. Finanzplatz-Informationen, 1/1998 (également dans Afrika-Bulletin, Febr./März 1998).

Mark Pieth, Peter Eigen (Hrsg.), Korruption im internationalen Geschäftsverkehr. Bestandesaufnahme Bekämpfung Prävention, Basel, 1998.

Handels Zeitung, «Liberalisierung schützt nicht vor Bestechung», 3.6.98; «Bestechung war lange Zeit ein Tabu», 14.9.98.

Informell, «Das schädliche schnelle Geld», 1/98.

Neue Zürcher Zeitung, «Korruption - ein korrupter Begriffs», «Bestechung in der Schweiz», «Bakschisch oder die permanente Umverteilung», 5-6.9.98.

Tages-Anzeiger, «Vorbeugen ist wichtiger als strafen» (entretien avec Mark Pieth), 13.7.98.

Blanchiment d'argent

«Loi fédérale concernant la lutte contre le blanchiment d'argent dans le secteur financier (Loi sur le blanchiment d'argent, LBA)», Feuille fédérale, nº 41 , vol. 4, 21.10.97.

«Loi fédérale sur les jeux de hasard et les maisons de jeu (Loi sur les maisons de jeu, LMJ)» du 18 décembre 1998, projet du Conseil fédéral du 26 février 1997, loi fédérale, Feuille fédérale, n 51, 30.12.98, pp. 5004 et suiv.

Financial Times, «Police Fear Euro-cash Will Bring Euro Crime», 14.7.98.

Handels Zeitung, «Schweiz unter der Lupe», 18.2.98; «Neue Offensive gegen Geldwäscher», 20.5.98; «Fast alle Schlupflöcher gestopft», 10.6.98.

La Liberté, «Une loi c'est bien. Mais collaborer avec l'étranger, c'est encore mieux», 27.3.98.

Neue Zürcher Zeitung, "Den Geldwäschern auf der Spur. Zunehmende Professionalität der FATF-Expertengruppe», 13.2.98; «Geldwäscherei-Gesetz tritt am 1. April in Kraft», 17.3.98; «Schweiz für organisierte Kriminalität attraktiv», 30.3.98; «Lukrativer Kampf gegen Geldwäscherei», 29.5.98; "Zahlreiche Meldungen über GeldwäschereiVerdacht», 10.7.98; «Was tun mit konfiszierten Drogengeldern?», 12.10.98.

Tages-Anzeiger, «Härtere Zeiten für Geldwäscher», 1.4.98.

Fuite de capitaux et entraide judiciaire internationale

Bern Declaration, The Swiss Business Links of the Suharto and Habibie Oligarchy of Indonesia. A Report by George J. Aditjondro, Newcastle University, Australia, 22.7.98.

Erklärung von Bern, Schweiz-Indonesien: Die Helfershelfer des Suharto-Regimes. Eine Studie über die wirtschaftlichen und politischen Beziehungen zwischen der Schweiz und dem Suharto-Regime, 22.7.98.

KEM-Info/Medien (Hrsg.), Mobutismus - Kalter Krieg und Plünder-Kumpanei. Beziehungen Schweiz-Kongo/Zaire von 1965 bis 1997, März 1998 (conception et rédaction de l'étude: Aktion Finanzplatz Schweiz).

Transparency Switzerland, Forum gegen Korruption. Schweizerische Rechtshilfe in Strafsachen. Eine Wegleitung zum Schweizer Bankgeheimnis, Juli 1998.

WochenZeitung, «Marcos-Gelder für Opfer. Bundesgericht gewichter Menschenrechte», 18.12.98.

\subsection{EXPORTATIONS D'ARMES ET POLITIQUE DE SÉCURITÉ DE LA SUISSE}

En 1997, le commerce des armes à l'échelle du globe a légèrement augmenté. L'Asie a cette année encore absorbé la moitié des exportations mondiales d'armes. Les fabricants suisses ont bénéficié pour la deuxième année consécutive d'une importante commande d'Oman, d'un montant de 61 millions de francs. L'année 1998 a été marquée en Suisse par l'entrée en vigueur de nouvelles lois régissant la possession et le commerce des armes. Au mois de septembre, la Confédération a signé deux importantes conventions internationales sur les essais nucléaires et les armes chimiques. Elle a renouvelé ses engagements en faveur du maintien de la paix en envoyant dans plusieurs pays des bérets bleus et des bérets jaunes. En ce qui concerne les mines antipersonnel, le Traité d'Ottawa, dont la Suisse fait partie, a pu entrer en vigueur grâce à la ratification rapide d'une cinquantaine d'Etats. Un Centre international de déminage humanitaire s'est ouvert à Genève, qui a pour objectif de renseigner et coordonner les diverses campagnes de déminage. 


\section{$\square$ Commerce international des armes}

Les exportations d'armes à l'échelle du globe ont augmenté de 22,54 milliards de dollars en 1996 à 25,16 milliards en $1997^{39}$. Les Etats-Unis sont restés le principal fournisseur d'armes conventionnelles avec $43 \%$ des exportations; ils sont suivis de la Russie (14\%), de la France (13\%) et de la Grande-Bretagne (10\%). En 1997, la moitié des ventes furent destinées à 1'Asie. Taiwan a importé pour 4 milliards de dollars de matériel militaire. La Chine, la Corée du Sud, l'Inde, la Thaïlande et la Malaisie ont aussi chacune investi plus d'un milliard de dollars dans des armes étrangères. Au Moyen-Orient, les principaux clients furent l'Arabie Saoudite (2,37 milliards de dollars) et la Turquie (1,28 milliard). A l'opposé, si l'on excepte les 0,87 milliard de dollars investis par l'Egypte, les achats de l'ensemble du continent africain se sont montés à 0,26 milliard de dollars.

\section{$\square$ Exportations suisses d'armement}

Les exportations suisses de matériel militaire en 1997 se sont élevées à un total de 294 millions de francs, soit 0,26\% de l'ensemble des marchandises exportées. Les principaux clients ont été Oman (61 millions), l'Allemagne (38 millions), les Etats-Unis (34 millions) et la Malaisie (27 millions). Les plus grosses transactions ont porté sur la vente de chars (Piranha). Dans le cadre des biens à double usage, l'essentiel des exportations fut constitué d'avions d'entraînement (type Pilatus PC7 et PC9).

Tableau $n^{\circ}$ 14: Exportations suisses de matériel de guerre 1996-1997* Les 15 principaux pays en développement et en transition clients de la Suisse

\begin{tabular}{|c|c|c|c|c|c|}
\hline \multicolumn{3}{|c|}{1996} & \multicolumn{3}{|c|}{1997} \\
\hline Pays** & $1000 \mathrm{fr}$. & en $\%$ & Pays** & $1000 \mathrm{fr}$. & en $\%$ \\
\hline Oman & 65426 & 28.1 & Oman & 61514 & 20.9 \\
\hline Inde & 5997 & 2.6 & Malaisie & 26977 & 9.1 \\
\hline Singapour & 5862 & 2.5 & Émirats arabes unis & 11230 & 3.8 \\
\hline Arabie Saoudite & 5472 & 2.3 & Bahreïn & 6803 & 2.3 \\
\hline Afrique du Sud & 3762 & 1.6 & Afrique du Sud & 6278 & 2.1 \\
\hline Pakistan & 2927 & 1.3 & Israël & 4306 & 1.5 \\
\hline Brésil & 2489 & 1.1 & Inde & 3527 & 1.2 \\
\hline Emirats arabes & 2398 & 1.0 & Arabie saoudite & 2280 & 0.8 \\
\hline Bahreïn & 1412 & 0.6 & République tchèque & 2051 & 0.7 \\
\hline Thaïlande & 1262 & 0.5 & Venezuela & 1489 & 0.5 \\
\hline Malaisie & 908 & 0.4 & Singapour & 1354 & 0.5 \\
\hline Egypte & 757 & 0.3 & Brésil & 1198 & 0.4 \\
\hline Corée du Sud & 735 & 0.3 & Chili & 1092 & 0.4 \\
\hline Tunisie & 704 & 0.3 & Pakistan & 890 & 0.3 \\
\hline |sraë| & 678 & 0.3 & Maroc & 826 & 0.3 \\
\hline $\begin{array}{l}\text { Autres pays en dévelop- } \\
\text { pement et en transition }\end{array}$ & 3565 & 1.5 & $\begin{array}{l}\text { Autres pays en dévelop- } \\
\text { pement et en transition }\end{array}$ & 4335 & 1.5 \\
\hline $\begin{array}{l}\text { Total pays en dévelop- } \\
\text { pement et en transition }\end{array}$ & 104354 & 44.8 & $\begin{array}{l}\text { Total pays en dévelop- } \\
\text { pement et en transition }\end{array}$ & 136148 & 46.3 \\
\hline Pays industrialisés & 128584 & 55.2 & Pays industrialisés & 158169 & 53.7 \\
\hline Exportations totales & 232938 & 100.0 & Exportations totales & 294317 & 100.0 \\
\hline
\end{tabular}

Source: Secrétariat général du DDPS, Ausfuhr von Kriegsmaterial 1997.

* Ne sont pas comprises dans ce tableau les exportations de biens à double usage.

* Pays de destination finale.

39. Stockholm International Peace Research Institute, Yearbook 1998, SIPRI, June 1998. Résumé des rapports SIPRI sur Internet: http://www.sipri.se (Les valeurs sont calculées en dollars à prix constants, avec pour base 1990.) 
Concernant les exportations d'armement, les entreprises suisses sont soumises à deux lois différentes: la loi sur le matériel de guerre et la loi sur le contrôle des biens à double usage.

ASTM 1997, pp. 106-107 (à propos de ces deux lois).

La Loi fédérale sur le matériel de guerre de 1996 (entrée en vigueur le $1^{\text {er }}$ avril 1998) met l'accent sur la nécessité de respecter les engagements internationaux et les principes de la politique étrangère de la Suisse. Ainsi, avant d'accorder une licence d'exportation de matériel de guerre, la Confédération veille au respect de certains points: maintien de la paix et de la sécurité internationale, respect des droits de l'homme, attitude du pays de destination envers la communauté internationale ${ }^{40}$. Cette loi soumet à autorisation non seulement la fabrication et le commerce de matériel de guerre, mais aussi le transfert de biens immatériels (technologie militaire, brevets). D'autre part, la loi interdit sur territoire suisse tout transfert et production d'armes nucléaires, biologiques ou chimiques. L'interdiction s'étend également à l'étranger pour toute personne suisse qui commettrait un acte contraire aux engagements internationaux de la Confédération.

La Loi fédérale sur le contrôle des biens à double usage et sur les biens militaires de 1996 (entrée en vigueur le $1^{\text {er }}$ octobre 1997) vise à contrôler le commerce des biens utilisables à la fois dans le domaine civil et militaire (tels que le matériel d'observation ou les masques à gaz). Par cette loi, la Suisse s'engage à respecter les embargos formulés par les Nations Unies et par l'Union européenne sur l'exportation des biens à double usage, pour autant que les principaux partenaires commerciaux de la Suisse appliquent aussi la directive. La liste des biens à double usage ${ }^{41}$ est calquée sur celle de l'UE. L'appellation «bien militaire spécifique» regroupe le matériel militaire qui n'est pas utilisé pour le combat. Les avions d'entraînement Pilatus PC en font partie.

\section{- Embargos appliqués par la Suisse en 1998}

\begin{tabular}{|c|c|}
\hline Afghanistan & armes et munitions à toutes les parties en conflit (ONU)*; \\
\hline Angola & $\begin{array}{l}\text { armement, pétrole et toute assistance militaire } \\
\text { destinés à l'UNITA - Union nationale pour l'indépendance } \\
\text { totale de l'Angola - (ONU); }\end{array}$ \\
\hline Birmanie & matériel de guerre (UE) ; \\
\hline Burundi & armes (ONU) : \\
\hline Géorgie & mines (ONU) ; \\
\hline Irak & armes et contrôle des fournitures humanitaires reçues (ONU) ; \\
\hline Liberia & armes et matériel militaire (ONU) ; \\
\hline Libye & armes, matériel et brevets y afférents (ONU) ; \\
\hline Nigeria & armes (ONU) ; \\
\hline Rwanda & $\begin{array}{l}\text { embargo sur les armes appliqué } \\
\text { aux forces non gouvernementales (ONU): }\end{array}$ \\
\hline Sierra Leone & armement et matériel connexe, produits pétroliers (ONU) ; \\
\hline Somalie & armes (ONU) ; \\
\hline Soudan & armes (ONU) ; \\
\hline Etats d'ex-Yougoslavie (tous) & matériel de guerre (UE). \\
\hline * Entre parenthèses est indique & lé qui a prononcé l'embargo. \\
\hline
\end{tabular}


Le 9 juin 1998, l'Union européenne a adopté un Code de conduite pour les exportations d'armes. Celui-ci oblige les membres de l'Union à tenir compte des sanctions et embargos décrétés par le Conseil de sécurité des Nations Unies, par l'OSCE ou par l'UE elle-même. Il interdit les exportations provoquant ou prolongeant un conflit armé ou des risques de conflit dans le pays de destination. Les pays exportateurs doivent veiller à ce que les équipements fournis ne soient pas transformés ou réexportés au profit d'un utilisateur non désiré. La Confédération ne s'est pas prononcée sur ce Code de conduite, celui-ci allant dans le sens de la législation déjà en vigueur en Suisse.

\section{$\square$ Orientations de la politique de sécurité de la Suisse}

Au mois de février 1998, la Commission d'étude pour les questions stratégiques a transmis son rapport (appelé généralement Rapport Brunner) au Conseil fédéral. Estimant que les risques ont changé de nature (prolifération des armes de destruction massive, terrorisme, migrations et réfugiés, menaces écologiques), la commission recommande de réorienter la politique de sécurité du pays. A l'avenir, la Suisse devrait, pour assurer sa protection, s'orienter vers d'avantage de coopération avec d'autres Etats (Union européenne surtout) et organisations internationales (CICR, ONU). Divers sondages montrent à ce propos que la population est de plus en plus favorable à l'engagement de troupes suisses volontaires auprès de l'ONU (66\% de la population d'après les études Sécurité 98 publiées par l'Ecole militaire supérieure, EPFZ). Actuellement, une vingtaine d'officiers suisses sont déjà engagés en qualité d'observateurs auprès de l'ONU (bérets bleus). Le Conseil fédéral a chargé un groupe de travail de mettre au point de nouveaux plans directeurs pour la politique de sécurité de la Suisse. Ce rapport, ainsi que la révision partielle de la loi sur l'armée, doivent être traités durant l'été 1999. Ces nouvelles mises à jour devraient permettre aux troupes suisses à l'étranger de s'armer pour leur propre protection.

Dans le cadre du Partenariat pour la paix (PPP) coordonné par l'OTAN, la Suisse a organisé cette dernière année 33 cours de formation en matière de politique de sécurité, de contrôle démocratique, de droit humanitaire et de maîtrise des armements. Ayant pour la majorité lieu au Centre de politique de sécurité (CPS) de Genève, ces cours sont principalement destinés aux diplomates, officiers et fonctionnaires des membres du PPP. Le partenariat a pour objectif de faire partager l'expérience internationale en matière de promotion de la paix et d'aide en cas de catastrophe. La participation aux actions du PPP n'étant pas obligatoire et systématique, le Conseil fédéral estime cet engagement compatible avec la politique de neutralité et le droit suisse. Les opposants à cette stratégie reprochent au gouvernement de ne pas avoir soumis de tels engagements internationaux au vote populaire. Ils font aussi remarquer qu'un pareil rapprochement avec l'OTAN n'est pas compatible avec la neutralité de la Suisse, encore souhaitée par $80 \%$ des citoyens. Enfin, ils mettent en cause la notion de maintien de la paix, lorsque celle-ci est assurée par une organisation avant tout militaire.

Cette dernière année, la Suisse a réaffirmé son soutien aux opérations de maintien de la paix de l'OSCE, en maintenant un contingent d'une cinquantaine d'opérateurs logistiques en Bosnie-Herzégovine. Entre les mois de décembre 1998 et janvier 1999, l'OSCE a déployé sa plus ambitieuse mission depuis sa création en envoyant 2000 volontaires au Kosovo. La Suisse a mis à la disposi- 
tion de l'organisation une centaine d'experts qui ont pour tâche de faire respecter le cessez-le-feu, lancer des négociations entre belligérants, soutenir des organisations humanitaires, aider le retour des réfugiés et à terme surveiller l'organisation des élections. Non armés, les bérets jaunes peuvent compter si besoin est sur le soutien d'une force d'intervention rapide de l'OTAN stationnée en Macédoine voisine.

\section{$\square$ Loi fédérale sur les armes, les accessoires d'armes et les munitions}

Cette loi, datée du 20 juin 1997, est entrée en vigueur le $1^{\text {er }}$ janvier 1999. Elle régit la fabrication, le commerce, les échanges et le port d'armes, d'éléments et d'accessoires d'armes ainsi que de munitions. Sont aussi considérés comme armes certains sprays d'autodéfense, les couteaux et poignards à lame escamotable et les appareils à électrochocs. Ceux-ci, avec les armes à feu automatiques et les accessoires tels que silencieux et viseurs lasers, sont interdits en Suisse. Les autres armes et les munitions peuvent être acquises moyennant un permis. Les fabricants et les commerçants doivent détenir une patente et répertorier leurs transactions. Les autorités de surveillance sont autorisées à pénétrer sans avis préalable dans leurs locaux commerciaux et peuvent consulter tous les documents utiles. Enfin, le Conseil fédéral peut interdire l'acquisition d'armes aux ressortissants de certains Etats s'il estime qu'il y a un risque d'utilisation abusive. (C'est le cas actuellement pour les ressortissants d'Albanie, d'Algérie, de Bosnie-Herzégovine, de Croatie, de Macédoine, de la République fédérale de Yougoslavie, de la Turquie et du Sri Lanka ${ }^{42}$.)

Le $1^{\text {er }}$ mai 1998 est entrée en vigueur l'Ordonnance concernant la transformation des rapports de service des entreprises d'armement de la Confédération. Celle-ci privatise les entreprises fédérales d'armement ${ }^{43}$, qui deviennent des sociétés anonymes de droit privé. La Confédération exerce dès lors ses droits en tant qu'actionnaire.

\section{$\square$ Nucléaire}

Le 9 septembre 1998, la Suisse a ratifié le Traité d'interdiction complète des essais nucléaires (TICE). Ce traité, négocié dans le cadre de la Conférence du désarmement, est une forme de complément au Traité de non-prolifération des armes nucléaires déjà existant. Signé par quelques 150 Etats, il n'entrera en vigueur que lors de la $44^{\mathrm{e}}$ ratification par un pays doté d'une capacité nucléaire. La Suisse fait partie de ces Etats en raison de la présence de réacteurs atomiques civils sur son territoire. L'objectif du traité est d'éviter la prolifération des armes nucléaires en empêchant les pays sans armement atomique d'en acquérir un et les puissances nucléaires déclarées de perfectionner le leur. Le TICE prévoit la mise en place d'un système international de surveillance basé à Vienne. Celui-ci vérifiera l'application du traité par les pays membres. Un réseau global de 321 stations de mesure recourra à quatre méthodes (sismique, hydroacoustique, infrasons, radionucléides) pour détecter d'éventuels essais.

42. Ordonnance du 21 septembre 1998 sur les armes, les accessoires d'armes et les munitions, art. 9.

43. Entreprises suisses d'aéronautique et de systèmes, de munitions, d'armement, d'électronique. 


\section{$\square$ Armes chimiques}

Le 11 septembre 1998, la Suisse a signé la Convention internationale régissant l'importation et l'exportation de produits chimiques (appelée Convention PIC). Cette convention a été préparée par le Programme des Nations Unies pour l'environnement et par l'Organisation des Nations Unies pour l'alimentation et l'agriculture. Elle a pour objectif d'empêcher que des produits chimiques dangereux ne soient exportés dans les Etats du Tiers Monde si la sécurité de leur maniement n'est pas assurée. La liste PIC recense actuellement 22 pesticides et 5 produits chimiques industriels qui ne pourront être exportés que si le gouvernement du pays importateur donne préalablement son accord. Le traité prévoit aussi d'améliorer l'information sur les risques que comporte le maniement de ces substances. Il deviendra obligatoire pour les Etats parties après la $50^{\mathrm{e}}$ ratification.

Avec l'entrée en vigueur (29 avril 1997) de la Convention sur les armes chimiques (CAC) la Suisse est tenue de déclarer la fabrication, le traitement et l'utilisation des substances dites à double usage (substances exploitées dans l'industrie chimique civile, mais aussi susceptibles d'être utilisées pour la production illicite d'armes chimiques). Dix entreprises suisses ont ainsi été sujettes à inspection en 1998. Le laboratoire AC de Spiez ${ }^{44}$ a par ailleurs été nommé «laboratoire de confiance» par l'Organisation pour l'interdiction des armes chimiques. Seuls sept laboratoires au monde ont jusqu'à présent reçu cette distinction. La convention CAC est ratifiée à ce jour par 116 pays. Les Etats parties se sont interdit la mise au point, la fabrication, le stockage et l'emploi d'armes chimiques. Ils se sont également engagés à détruire leurs stocks et installations de production de ces armes.

\section{$\square$ Mines antipersonnel \\ ㅁ. ASTM 1998, pp. 258-260.}

Le Traité d'Ottawa sur les mines antipersonnel est entré en vigueur le $1^{\text {er }}$ mars 1999, la barre des 40 ratifications nécessaires ayant été atteinte durant l'automne 1998. L'extrême rapidité avec laquelle ce traité a rejoint le droit international résulte d'une bonne collaboration entre les gouvernements et les ONG concernées, ainsi que d'une importante mobilisation au sein du public contre les mines

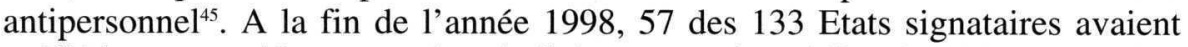
ratifié le texte et 12 pays - dont la Suisse - avaient déjà achevé la destruction totale de leurs stocks ${ }^{46}$. Néanmoins, malgré ce large ralliement, certains pays restent opposés à tout engagement international. Parmi la soixantaine de pays n'ayant pas signé le document figurent des pays importants tant militairement que politiquement comme les Etats-Unis, la Russie, la Chine, l'Inde ou l'Egypte. Ces pays non seulement conservent le droit d'utiliser des mines antipersonnel, mais peuvent aussi continuer de les fabriquer et de les exporter. La disponibilité de ces armes sur le marché international pose problème à certains Etats qui, tout

44. Le laboratoire AC de Spiez est une branche du Groupement pour l'armement du DDPS. Actif dans le domaine du contrôle des armes atomiques, biologiques et chimiques, le laboratoire a été mandaté à plusieurs reprises par l'ONU pour analyser des échantillons provenant de zones en conflit.

45. Rappelons que les estimations font état de quelque 110 millions de mines disséminées dans une septantaine de pays, causant la mort de 800 personnes et en mutilant 1200 autres chaque mois - les trois quarts des victimes étant des enfants.

46. On trouvera une liste des adhérents au traité régulièrement mise à jour sur le site Internet suivant: http://www.handicap-international.org 
en ayant ratifié le Traité d'Ottawa, sont confrontés à la pose de mines sur leur territoire par des groupes rebelles. Ce fut le cas de l'Angola et de la Colombie en $1998^{47}$.

Durant cette dernière année, un nombre important de conférences sur les mines et le déminage ont été organisées (conférences de Budapest, Moscou, Washington, Phnom Penh, Tokyo, Edinburgh, etc.). De portée régionale ou internationale, ces réunions ont permis à des représentants de gouvernements et d'ONG ainsi qu'à des experts du déminage de coordonner leurs efforts. 1998 a été marquée en Suisse par l'inauguration, le 28 avril à Genève, du Centre international de déminage humanitaire (CIDH). Installé depuis février 1999 dans le nouveau bâtiment de l'Organisation mondiale de météorologie, le centre emploie en permanence une quinzaine de personnes. Dix-sept pays ont déjà manifesté leur collaboration et leur soutien. L'activité du CIDH se déploie dans quatre directions principales:

- cellule de réflexion permanente animée par des experts, chargée d'analyser les expériences réalisées sur les différents sites de déminage, d'identifier les problèmes et de proposer des solutions sous forme de normes ou de méthodes;

口 système de gestion de l'information adapté aux besoins des Nations Unies et des divers centres d'action contre les mines;

- organisation de conférences périodiques permettant aux responsables des opérations de déminage d'échanger leurs expériences;

- organisation, à Genève et sur le terrain, de cours de formation pour les futurs responsables d'opérations de déminage ainsi que pour les spécialistes de la gestion de l'information ${ }^{48}$.

La Fédération suisse de déminage a pu déployer son action sur le terrain bosniaque en 1998, les 200'000 francs nécessaires pour mener à bien sa première opération ayant été réunis. Le Département des affaires étrangères a financé le projet à hauteur de $60 \%$. Cette organisation, créée en juillet 1997 par des anciens du CICR et de l'Action humanitaire, entend continuer de rechercher des fonds pour étendre son action dans d'autres pays, notamment au Nicaragua. Le déminage est une opération très coûteuse: selon des estimations des Nations Unies, une mine terrestre, qui à l'achat vaut 3 dollars et dont la pause ne coûte pratiquement rien, revient entre 200 et 1000 dollars au moment de l'enlever ${ }^{50}$. En effet, ce n'est pas le nombre de mines neutralisées qui est important, mais le nombre de mètres carrés nettoyés. Une opération de déminage se déroule en plusieurs phases: il faut tout d'abord repérer et cartographier les zones minées, puis détecter et neutraliser les explosifs avant de pouvoir les détruire. Parallèlement, des programmes de prévention sont mis en place et des démineurs locaux sont recrutés. Le déminage manuel reste actuellement encore la méthode la plus fiable. En garantie supplémentaire, une fois une parcelle déminée, on fait généralement appel à des chiens dressés pour détecter les odeurs de poudre explosive. De nombreuses recherches sont en cours pour développer des moyens plus

47. Selon Elisabeth Reusse-Decrey, coordinatrice de la Campagne suisse contre les mines antipersonnel.

48. Tiré du discours inaugural prononcé le 28 avril 1998 par le directeur du CIDH, François Godet.

49. Le DFAE a aussi décidé verser plus de 400'000 francs à l'organisation Handicap International pour son action de déminage en Bosnie.

50. Estimation faite par les Nations Unies, citée dans Le Courrier du 29.9.98, p. 16. 
rapides et plus sûrs de déminer. Un de ces procédés se base sur les ondes infrarouges: les matériaux utilisés dans la fabrication des mines étant différents des composants du sol, leur écart de température pourrait être détecté moyennant des appareils suffisamment sensibles. Utilisant aussi les ondes, une autre étude cherche à identifier la fréquence de résonance magnétique des matières explosives. Enfin, un laboratoire du Massachusetts a développé des polymères fluorescents, dont le rayonnement lumineux s'affaiblit considérablement au contact de vapeurs de TNT $^{51}$. Si le nouveau centre (CIDH) de Genève obtient une audience internationale suffisante, il pourra à l'avenir coordonner ces diverses recherches et informer les organisations de déminage des nouveaux procédés disponibles.

\section{Conventions internationales sur le désarmement dont la Suisse fait partie (date de ratification)}

口 Convention du 10 décembre 1976 sur l'interdiction d'utiliser des techniques de modification de l'environnement à des fins militaires ou toutes autres fins hostiles (5.8.88).

口 Convention du 10 octobre 1980 sur l'interdiction ou la limitation de l'emploi de certaines armes classiques qui peuvent être considérées comme produisant des effets traumatiques excessifs ou comme frappant sans discrimination (20.8.82). Protocole additionnel du 13 octobre 1995 sur les armes à laser aveuglantes (24.3.98). Protocole additionnel révisé le 3 mai 1996 sur l'interdiction ou la limitation de l'emploi des mines, pièges et autres dispositifs (24.3.98).

- Convention du 13 janvier 1993 sur l'interdiction de la mise au point, de la fabrication, du stockage et de l'emploi des armes chimiques et sur leur destruction (10.3.95).

口 Traité du 10 septembre 1996 sur l'interdiction complète des essais nucléaires.

- Convention du 3 décembre 1997 sur l'interdiction de l'emploi, du stockage, de la production et du transfert des mines antipersonnel et sur leur destruction (24.3.98).

Source: Feuille fédérale, nº 46, 24.11.98.

\section{SOURCES}

«Convention sur l'interdiction de la mise au point [...] des armes chimiques et sur leur destruction», Recueil officiel des lois fédérales, $\mathrm{n}^{\circ} 5,10.2 .98$, pp. 335-476.

«Loi fédérale sur le matériel de guerre» et «Ordonnance portant [...] sur le matériel de guerre», Recueil officiel des lois fédérales, n9, 10.3.98, pp. 794-806 et 807-825.

«Loi fédérale sur les biens utilisables à des fins civiles et militaires et des biens militaires spécifiques» et «Ordonnance sur l'exportation, l'importation et le transit [...] biens militaires spécifiques», Recueil officiel des lois fédérales, n $33,2.9 .97$, pp. $1697-1703$ et $1704-1712$.

«Message concernant la Convention sur l'interdiction [...] des mines antipersonnel et sur leur destruction» et «Convention sur l'interdiction [...] des mines antipersonnel», Feuille fédérale, nº 8, 3.3.98, pp. 537-556 et 559-571.

"Mines antipersonnel. Interdiction. Convention», Bulletin officiel de l'Assemblée fédérale. Session de printemps 1998, Conseil des Etats, pp. 194-196, et Conseil national, pp. 370-372.

«Ordonnance concernant la transformation des rapports de service des entreprises d'armement de la Confédération» du 25 mars 1998, Recueil officiel des lois fédérales, ${ }^{\circ} 18,12.5 .98$, pp. 1426-1428.

DDPS, La neutralité de la Suisse, DDPS, Berne, 1998 ( $\mathrm{n}^{\circ}$ 95.630).

Le Courrier, «Le Partenariat pour la paix de l'OTAN ne servira ni la paix, ni la Suisse», 6.2.98; «La Fédération suisse de déminage passe aux actes à Dobrinja», «La paix, l'armée suisse de demain devra la défendre à l'étranger», «Que la neutralité montre son utilité», 27.2.98; «Armes légères, destructions massives», 5.8.98; «Vingt-sept pays ont interdit les armes à laser aveuglantes», 10.8.98; «Le Conseil fédéral veut faire bannir les essais nucléaires», 11.9.98; «Ils arrachent les mines une à une», 29.9.98; «L'action suisse contre les mines vise les Etats réticents», 4.12.98.

Le Temps, «Armes biologiques: Genève souhaite accueillir les inspecteurs internationaux», 21.3.98; «La Suisse doit liquider 100'000 tonnes de munitions», 27.3.98; «La Suisse veut être le carrefour mondial de l'information sur les mines», 29.4.98; «La Suisse veut accueillir une conférence de l'ONU», 19.8.98.

Neue Zürcher Zeitung, «Suche nach neuen Technologien zur Beseitigung von Minen», 3.11.98.

\section{SITES INTERNET}

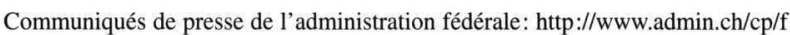

Sur les mines antipersonnel: http://www.handicap-international.org et http://www.icbl.org 